\title{
Plasma texturing of polymers
}

Jean-François Coulon ${ }^{1,2)}$, Dominique Debarnot ${ }^{2)}$, Fabienne Poncin-Epaillard ${ }^{2)}$

1) Ecam Rennes - Louis de Broglie, Campus de Ker Lann, CS 29 128, 35091 Rennes Cedex 09, France

2) Le Mans Université - CNRS n6283, Institut des Molécules et Matériaux du Mans Département Polymères, Colloïdes et Interfaces, Avenue Olivier Messiaen, 72085 Le Mans, France

Table of contents

Abstract

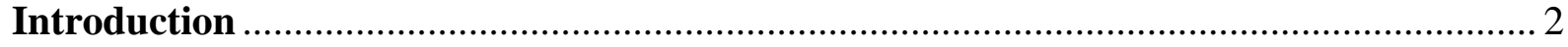

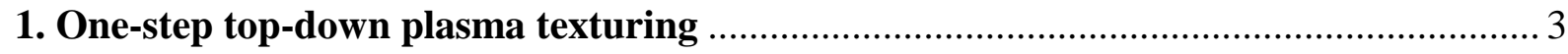

1.1 Control of the texture thanks to the plasma properties....................................................... 3

1.2 Control of the texture thanks to the polymer properties ................................................... 9

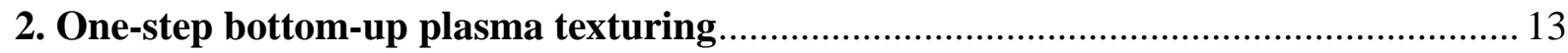

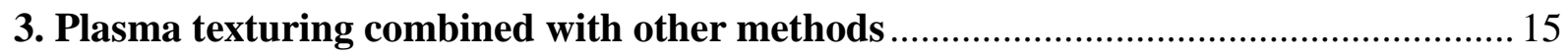

3.1 Plasma texturing combined with laser irradiation ........................................................... 15

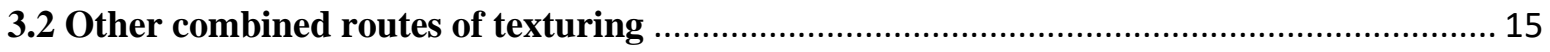

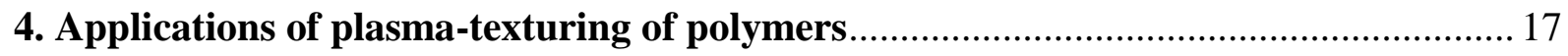

4.1 Application of plasma-texturing of polymers for the optic and electronic fields ................ 18

4.2 Application of plasma-texturing of polymers for the sensor field...................................... 19

4.3 Application of plasma-texturing of polymers for the biomedical field ............................... 21

4.4 Application of plasma-texturing of polymers for the metal adhesion ................................. 23

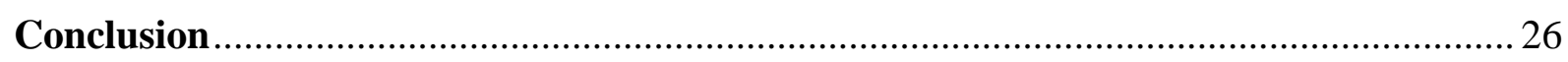

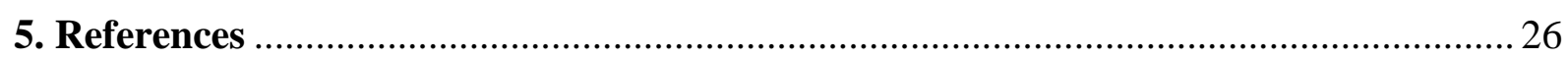




\begin{abstract}
This review explores the different processes usually applied in the field of nanotechnology for texturing polymeric materials. These technics mostly focused on dry and eco-friendly routes allow to create a multitude of patterns on the polymer surfaces bringing to the material specific and unique properties. Among these technics, plasma chemistry explored alone or combined with lased, beam, sputtering technologies... appears to be well adapted to thermosensitive materials for the preparation of original textured surfaces close to biomimetic surfaces as an example. This overview focuses as well as on the fundamental aspect of the texture creation than to its application in various industrial domains.
\end{abstract}

\title{
Introduction
}

Nanoscience and nanotechnology related to microelectronics, have brought about considerable new applications in the field of materials. Indeed, the change of scale passing from the micrometer to the nanometer has strong consequences on the final properties of a material widening the industrial fields and it could even open up new sectors of applications. These materials with enhanced properties are mostly based on the duplication of a pattern on double or multiple scales. This concept is originated from the observation of nature and more generally on biomimetism. Indeed, the nature offers many examples combining adapted surface chemistry and repeating pattern which give rise to remarkable properties like the water drop repelling, the ability to change the light reflecting or some extraordinary hooks on vertical or movable surfaces. The development of nanotechnology has made it possible to replicate the patterns observed in nature, hence texturing is the process which let us obtain a regular pattern on a surface.

Among the procedures deployed for texturing, two main routes are described. The first known as top-down for which the localized removal of material makes it possible to provide the pattern while the second known as bottom-up provides a textured material according to a precise topographic diagram.

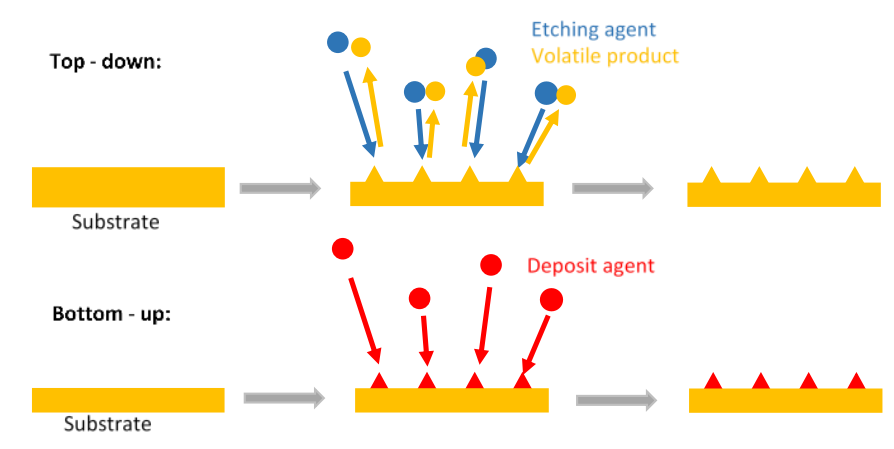


The surface chemistry can also contribute and can be simultaneous with this texturing step. Few processes allow both mechanisms and most of them require two or more steps to introduce expected texture and chemistry; some preparations also include the use of masks. An exception is focused on plasma technologies. In this chapter, we will describe how we can texture a polymer surface during its functionalization in one or two steps thanks to the plasma chemistry.

\section{One-step top-down plasma texturing}

Micro, nano, double scale-texturing of polymers offers the possibility to drive important surface properties such as wettability, (bio)adhesion or anti-(bio)adhesion applied in various domains such as medical devices, biology, metalization, optics and electronics. In recent years, plasma-induced self-assembly of polymer nanostructures without any mask has emerged as a simple, scalable and rapid etching technique to pattern micro and nanostructures. This elaboration of polymer nanofiber arrays is correlated to plasma etching and the nanostructures are dependent on both plasma parameters and polymer characteristics. The general statement concerning the nanofeatures growth claims that the selectivity in etching is due to the difference of the density through the material, intrinsic for a given material.

\subsection{Control of the texture thanks to the plasma properties}

The plasma parameters act upon the reactive species nature, their relative concentration in the plasma and upon the associated energy deposited on the surface which has to be modified. Basically, the efficient plasma parameters are the gas pressure, the gas flow rate and the chemistry of the input gas composition as well as the power, the duration and the frequency of the discharge. The literature reports on almost all types of polymers (PC, PMMA, PET, PEEK, PS, silicone, elastomers ...) which have been plasma-modified to present textured surface when subjected to different plasma phases (mainly $\mathrm{O}_{2}, \mathrm{CF}_{4}$, Ar and their mixtures....) [1-19]. The subsequent pattern is depending on plasma parameters. As an example, the higher the energy of the plasma is (like the duration or the power of the discharge), the more numerous and deeper the surface features are. Indeed, the PET when directly treated by an oxygen plasma shows a nano-fibrillar pattern [2] which density depends on the duration and on the discharge power. Without requiring masks, this route induces a similar surface topography than that one obtained 
with a filter in a two-step method, i.e. an optical lithography step followed by a plasma etching or a plasma deposition step [4]. The SEM pictures (Fig. 1) show that the density and the dimensions of the nanofibers increase with hard plasma conditions as the power. However, if the plasma parameters are too drastic (here $200 \mathrm{~W}$ ), the fibers collapse. Such a behavior is significant of the fiber growth mechanism corresponding to local melting of polymeric chains, crystallization and aggregation of oxidized PET oligomers. These oxidized oligomers are repulsed by the untreated sub-layer chains because of their more hydrophilic character. The hydrophilic character can be prevented if such a surface is then plasma-fluorinated to getdepending on the plasma parameters- the true Cassie-Baxter surface $\left(\Theta_{\mathrm{H} 2 \mathrm{O}}=157 \pm 2^{\circ}, \mathrm{H}_{\Theta}=\right.$ $1.6 \pm 0.6^{\circ}$, mean distance between two features $\approx 70 \mathrm{~nm}$ ) or the sticky superhydrophobic surface $\left(\Theta_{\mathrm{H} 2 \mathrm{O}}=156 \pm 5^{\circ}, \mathrm{H} \Theta=65 \pm 4^{\circ}\right.$, mean distance between two features $\left.\approx 145 \mathrm{~nm}\right)$ as also noticed in [20].
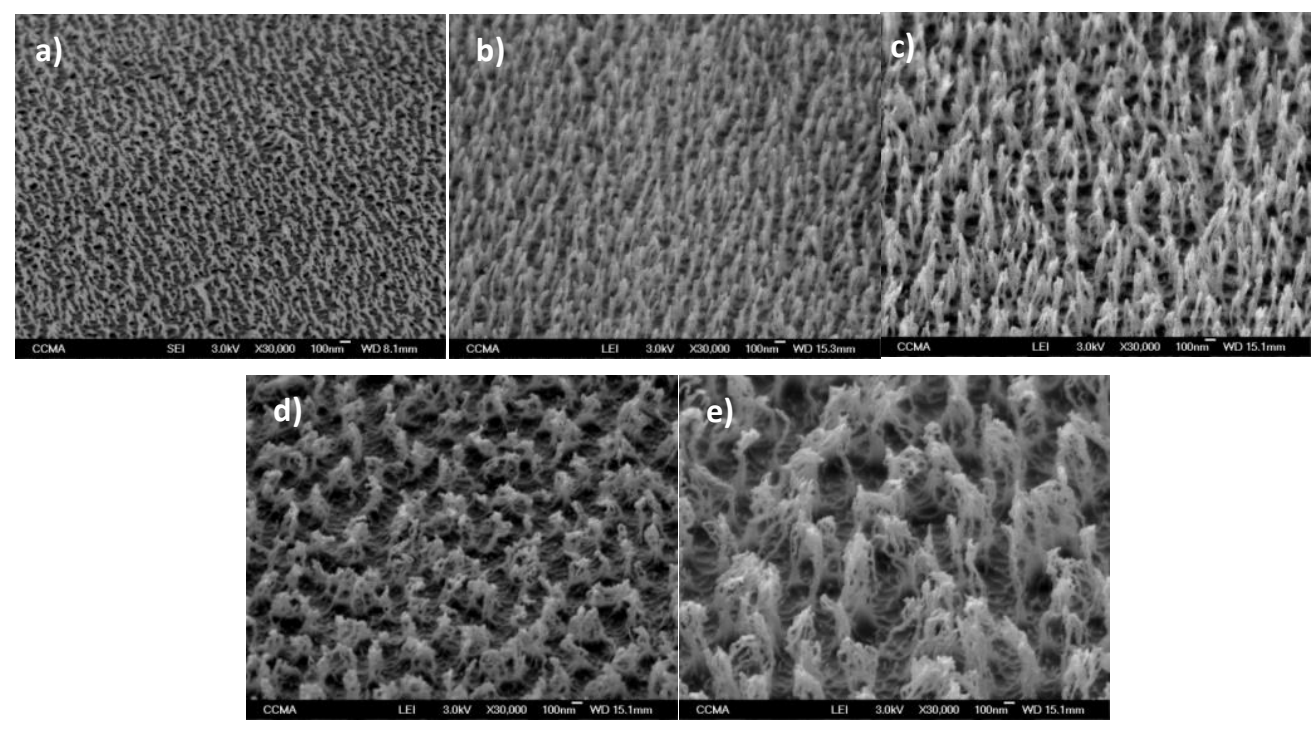

Fig. 1. Dependence of the texturing of PET on $\mathrm{O}_{2}$ plasma parameters [2]

$\left(P=70 \mathrm{~W}, p=0.02\right.$ torr, $t=15 \mathrm{~min}, F O_{2}=15 \mathrm{sccm}(\mathrm{a}), 50 \mathrm{sccm}(\mathrm{b}) ; P=100 \mathrm{~W}, p=0.02$ torr, $t=15 \mathrm{~min}, F O_{2}=$ $50 \mathrm{sccm}(\mathrm{c}) ; \mathrm{FO}_{2}=50 \mathrm{sccm}, p=0.08$ torr, $t=15 \mathrm{~min}, P=100 \mathrm{~W}(\mathrm{~d}), 200 \mathrm{~W}$ (e)

Similar etching plasma phases fed with $\mathrm{CF}_{4} / \mathrm{O}_{2}$ mixtures [7] allow to achieve superhydrophobic and slippery surfaces whose morphology and distribution are plasmadependent. The denser the plasma phase is, the higher the nanofeatures height is. However, their density is decreasing leading to a progressive reduction of the Cassie-Baxter character. The fluorinated polymers also lead to patterned surfaces in specific plasma conditions, whose micro and nano-features exhibit a pyramidal shape with a well-finished spherule on the apex [15]. At lower RF power and process time, only pyramids can be observed. Their creation was 
explained by the deposition onto the surface of a cluster of metal sputtered from the electrodes, building up a nano-mask followed by an aggregation (filaments, oblate forms, bead shapes) of the polymeric species and the metal atoms (Fig. 2).
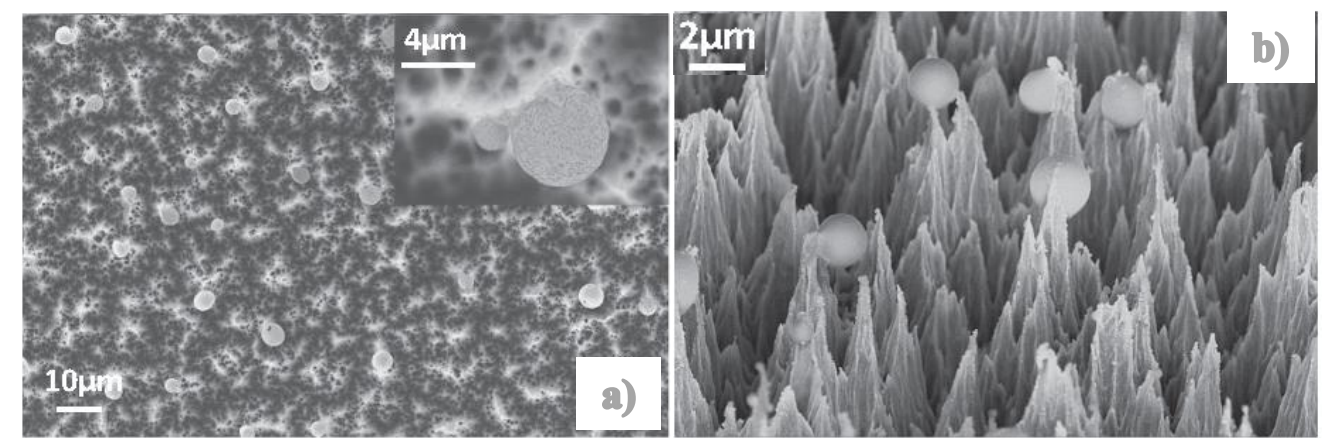

Fig. 2. Plasma-etched PTFE $\left(\mathrm{O}_{2}\right.$ plasma (a): $t=30 \mathrm{~min}, P=300 \mathrm{~W}$; (b) tilted of $45^{\circ}$, magnification $10000 \mathrm{x}, t=$ $30 \mathrm{~min}, P=200 \mathrm{~W}$ ) [15]

If the duration and the power of the plasma discharge increase, the surface temperature can be locally high enough to soften the matter and to reorganize the surface structure. However, the obtained surface topography is very close to that one obtained by a simple thermal annealing of PTFE. Such surface topography alteration has to be interpreted rather as a low effect of selfmasking from the incorporated metallic cluster than as a crystallite growth and a stress relaxation of the PFTE [21] (Fig. 3). In the case of the thermal annealing of PTFE, the annealing time above the melting temperature $(\mathrm{Tm})$ and the cooling time between $\mathrm{Tm}$ and the glass transition $(\mathrm{Tg})$ could both govern the creation of two types of texturation leading to a superhydrophobic state.
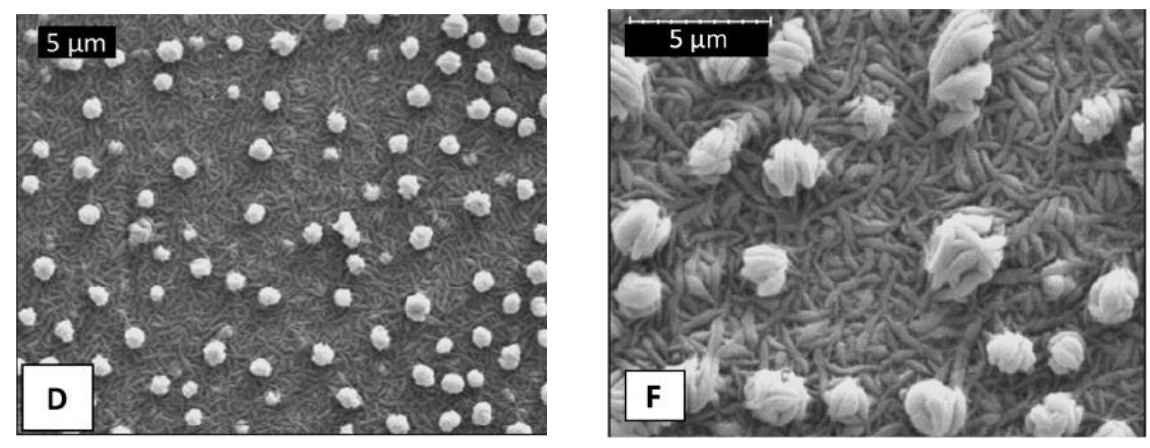

Fig. 3. Thermal texturing of PTFE with two patterns d) wart creation depending on the annealing time at $350^{\circ} \mathrm{C}$ f) dendrite creation depending on the cooling time between $\mathrm{Tm}$ and $\mathrm{Tg}$ [21]

To summarize, depending on the plasma parameters mostly the power and the duration of the discharge, the pattern of various polymers (thermoplastic, thermoset, elastomer, polysiloxane) can tune the hierarchical surface topography at different scales ( $\mu \mathrm{m}, \mathrm{nm}$ and both of them). However, the measured roughness is usually described as a mean value that is much too restrictive in order to provide the understanding of the subsequent specific surface behavior (either superhydrophobic or superhydrophilic). In order to separate out the roughness double 
scale, Fast Fourier Transform filtering analysis based on scaling theory was performed on AFM images of $\mathrm{CF}_{4}$ plasma-textured polybutadiene [22]. Using this approach, the micro and nano roughness were respectively determined and their summation matches well with the average roughness value usually determined for such a double-scale surface. Besides, as expected, although all three morphologies are hydrophobic, only the double-scale composite surface displays a low contact-angle hysteresis thus making it superhydrophobic surface.

The double-scale pattern was not observed for PDMS whatever the plasma conditions. Indeed, the regular double-scale pattern is replaced by a wrinkling design with a high nanoscale amplitude and with a periodicity of about $100 \mathrm{~nm}$ [1]. Increasing the plasma treatment duration, the roughness increases while the periodicity decreases until reaching a plateau, resulting in an enhanced surface area. Such behavior was interpreted as a consequence of the thin silica-like layer creation at the PDMS top surface and its strain due to simultaneous ionenhanced etching, oxidation during $\mathrm{O}_{2}$ plasma treatment (Fig. 4).
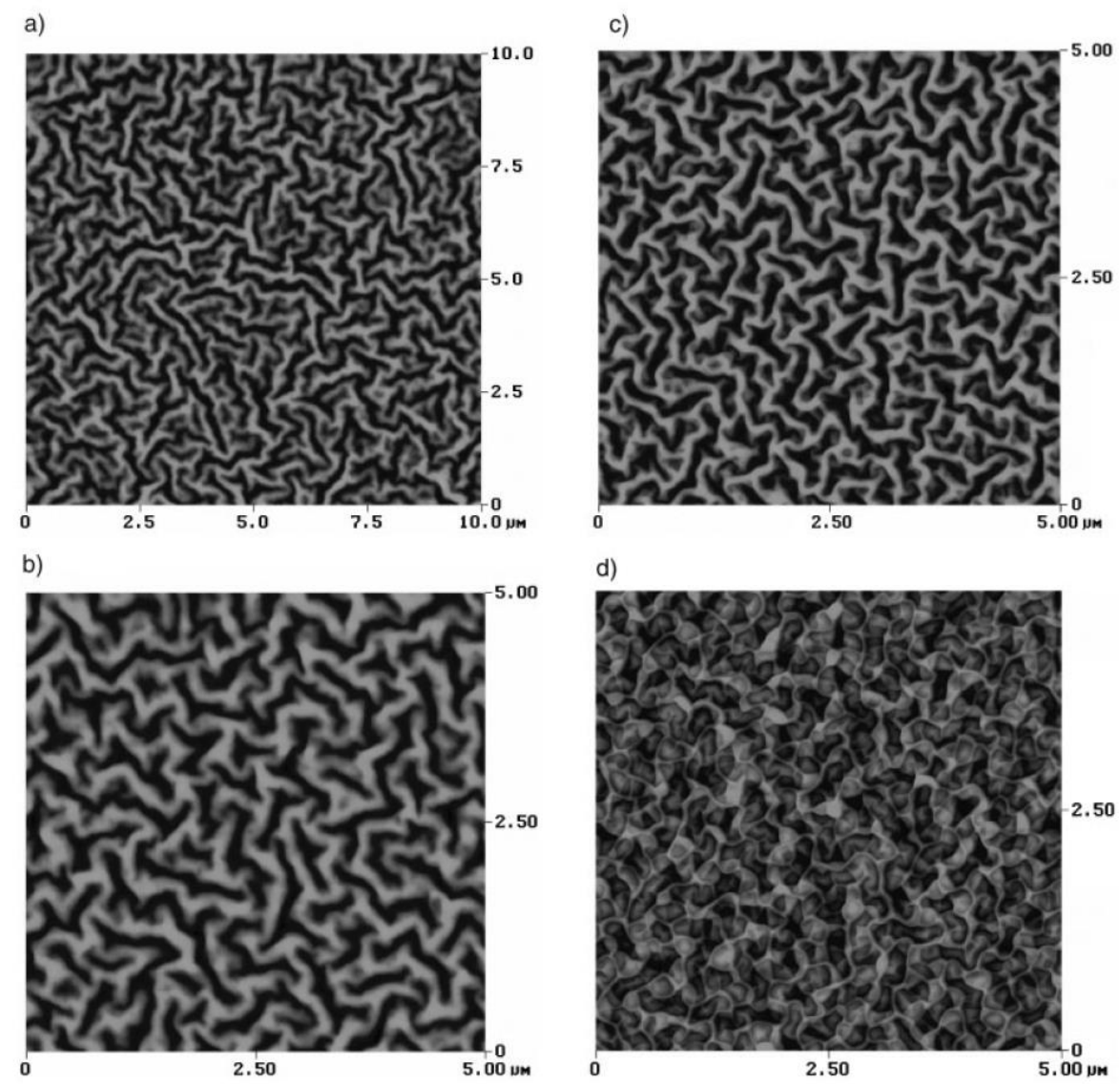

Fig. 4. AFM images of PDMS treated in a RIE O 2 plasma for (a) $30 \mathrm{~s}$, (b) $60 \mathrm{~s}$, (c) $420 \mathrm{~s}$ and (d) $600 \mathrm{~s}$. Roughness (rms) and periodicity ( $\lambda$ ) values for each surface are as follows: (a) $\mathrm{rms}=23 \mathrm{~nm}, \lambda=579 \mathrm{~nm}$, (b) rms $=46 \mathrm{~nm}, \lambda=338 \mathrm{~nm}$, (c) $\mathrm{rms}=37 \mathrm{~nm}, \lambda=366 \mathrm{~nm},(\mathrm{~d}) \mathrm{rms}=28 \mathrm{~nm}, \lambda=461,331,149,133 \mathrm{~nm}$. [1]

The plasma gas composition is well known to produce a large variety of interactions in the gas phase and at the interface of any solid material. Depending on the chemical nature of 
the gas phase, these reactions are classified as chemical or physical ones. At a first glance, the former generates new functional groups at the material surface while the latter leads to higher energy brought to the surface. This higher energy can lead to sputtering processes which may induce the texturation. One would assume that the texturing of polymeric surface requires only physical interactions, and therefore neutral gases such as argon could be sufficient. However, the appearance of a pattern is not only synonymous of ablated matter. As well as the physical interactions between plasma and surface can also lead to surface chemical modifications, the chemical interactions between plasma and surface can give birth to topographical modifications too. Thus, the achieved pattern in the case of polymers could also proceed via aggregation, rearrangement, chain reptation or functional groups turn over. These polymer chemistry phenomena are enhanced by the chemical affinity and, therefore reactive plasmas can also produce patterns on polymeric surfaces [1,3,20,23-27]. Furthermore, whatever the plasma chemical composition $\left(\mathrm{O}_{2}\right.$ or $\left.\mathrm{Ar}\right)$, the same trend for the roughness and for the periodicity of the texture for a plasma-treated PDMS surface is observed [3]. The only difference is noticed with a maximum value which occurs at $2 \mathrm{~min}$ for $\mathrm{Ar}$ treatment against $7 \mathrm{~min}$ for $\mathrm{O}_{2}$ plasma [1]. This result may be surprising since both plasma phases have different chemistry; the Ar plasma mostly induces sputtering while the latter one mostly produces the oxidation. This may suggest that the PDMS surface properties are prevalent rather than the plasma properties. In an opposite manner, if $\mathrm{O}_{2}$ and $\mathrm{CF}_{4}$ plasma phases strongly induce a chemical process, it appears that on $\mathrm{PC}$, the $\mathrm{CF}_{4}$-to- $\mathrm{O}_{2}$ feed ratio significantly alters the shape and the distribution of the generated structures. $\mathrm{O}_{2}$ plasma leads to taller structures with wire-like aspect and more homogeneous distribution. The duration as observed previously alters mainly the dimension [23]. Besides the competitive reactions of surface functionalization and degradation, the interactions between plasma species and polymeric surface induce cross-linking reactions in inert atmosphere such as Ar or He with a subsequent mechanical stress appearing between cross-linked surface and the bulk matrix. And if after the long Ar plasma treatment (30 min), the polymeric film is kept under high pressure and temperature, that causes the creation of multiscale hierarchical wrinkles with nano-textured features [26] (Fig. 5.).

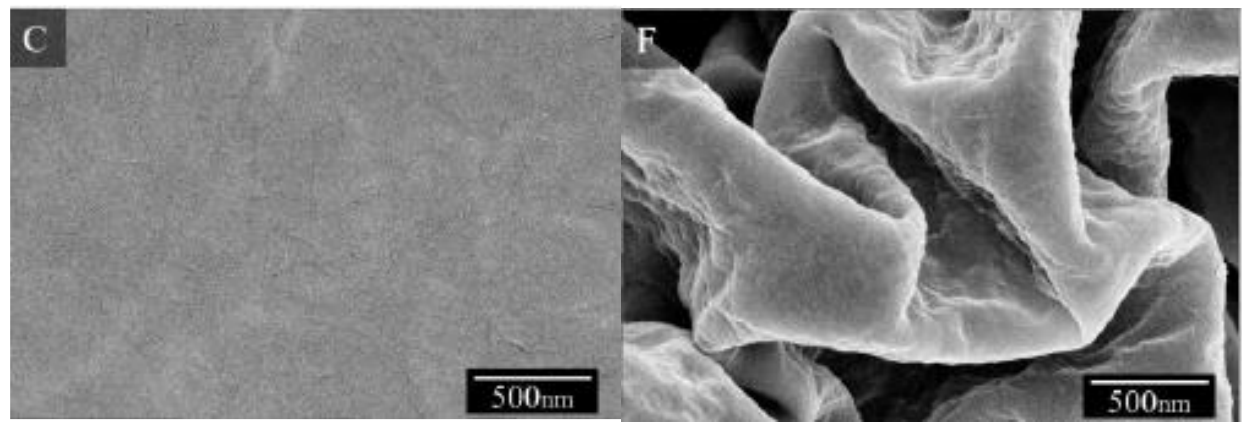




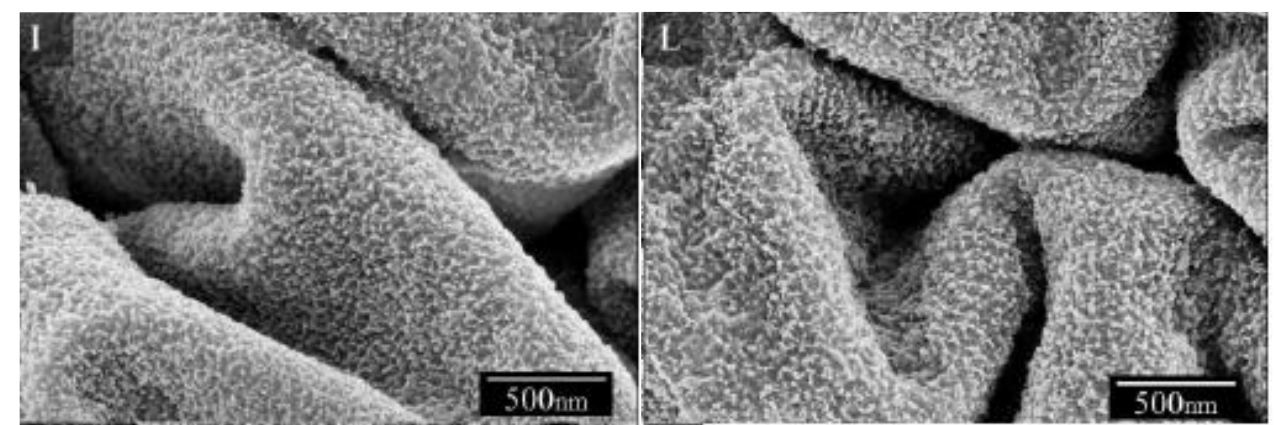

Fig. 5. SEM pictures of shrunk Ar plasma-treated polyethylene/polypropylene blend (c: blank; F: 1 min, I: 30 $\min , \mathrm{L}: 60 \mathrm{~min}$ ) [26]

The sputtering reaction can be emphasized by applying a bias voltage onto the substrate during the plasma exposure, whatever the excitation mode and the other plasma parameters. Such an experimental procedure significantly induces an anisotropic ion flux towards the surface to be treated $[24,25]$. Without any external bias applied to the substrate, the texturation pattern does not appear on the plasma treated PEEK surfaces [24] or is less pronounced in the case of fluorinated poly(ethylene propylene) (FEP) showing rounded peaks, synonymous with few degradation and texturation [25]. When a bias voltage is applied, the surface texture is observed in both cases and can be reinforced by adding reactive gases such dioxygen or even dinitrogen (Fig. 6). Furthermore, fluorinated polymer shows sharp features leading to a CassieBaxter surface (high WCA and high CA hysteris) while the rounded form belongs to the wenzel type surface if we consider only the classical root mean square average RMS [2].
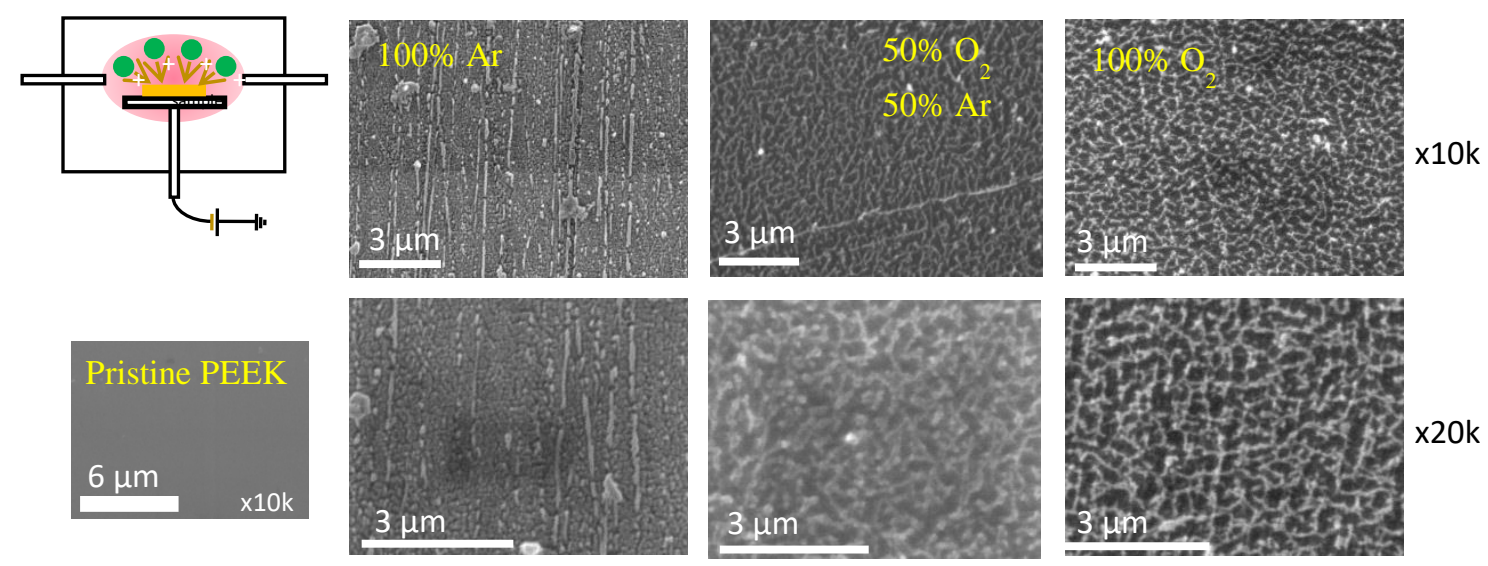

$x 20 k$

Fig. 6. SEM pictures of plasma-treated PEEK with a bias voltage of - $200 \mathrm{~V}$.

$\left(\mathrm{Ar} / \mathrm{O}_{2} P=1500 \mathrm{~W}, t=15 \mathrm{~min}, F=10 \mathrm{sccm}\right)$ [24]

Atomic force microscopy reveals the dimensions of these features (Fig. 7). With pure Ar plasma, the topography of the surface is governed by surface ripples which provide regular lines spaced by one or two hundred nanometers and up to 100 nanometers height. This kind of texturing is assumed to be mainly due to a thermal macroscopic rearrangement of the surface 
following the high ion energy impacting the surface. In such plasma conditions, one has to take care not to extand too much the bias to avoid the surface degradation of the polymer. With pure dioxygen, an isotropic pattern is thereby well defined and composed of homogenous but quite separated features of about tens of nanometer height. This texturation is correlated to different etching mechanisms of the polymer due to its difference in crystallinity.

a)

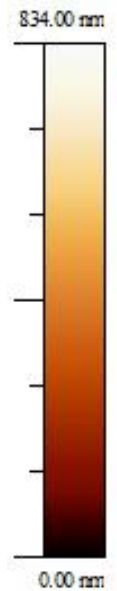

b)

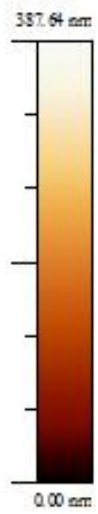

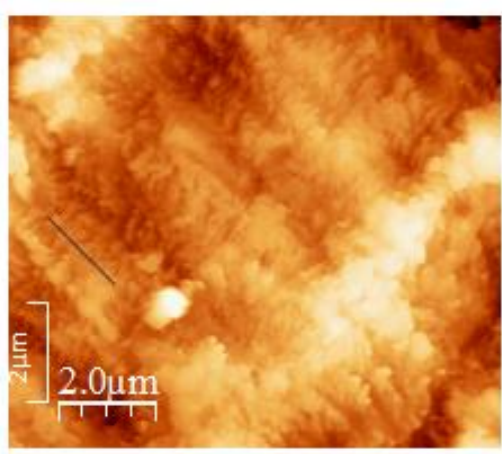

Image $10 \mu \mathrm{m} \times 8 \mu \mathrm{m}$

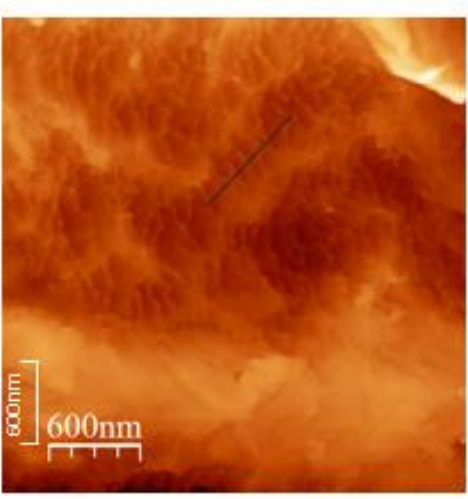

Image $3 \mu \mathrm{m} \times 3 \mu \mathrm{m}$
$100 \% \mathrm{Ar}$ $t=30 \min , P=1500 \mathrm{~W}, U=-100 \mathrm{~V}$

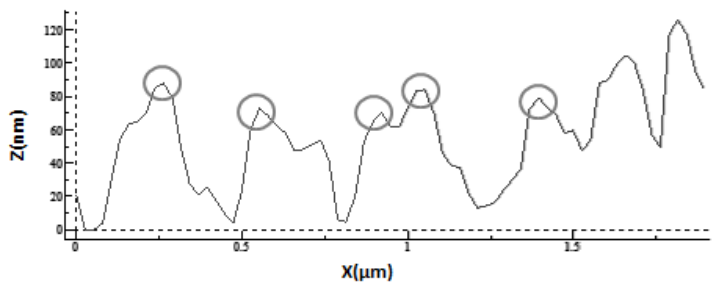

Height: $81.6 \pm 1.5 \mathrm{~nm}$

Width : $171.7 \pm 3.9 \mathrm{~nm}$

$100 \% \mathrm{O}_{2}$ $t=15 \min , P=1500 \mathrm{~W}, U=-100 \mathrm{~V}$

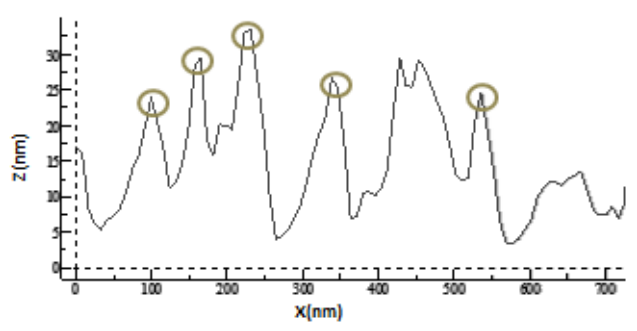

Height: $26.0 \pm 1.0 \mathrm{~nm}$ Width: $68.2 \pm 1.5 \mathrm{~nm}$

Fig. 7. AFM images and profiles for a) pure $\mathrm{Ar}$ and b) pure $\mathrm{O}_{2}$ plasma-treated PEEK -Bias voltage of - $100 \mathrm{~V}$ [28]

\subsection{Control of the texture thanks to the polymer properties}

The first property of a polymer to be textured is its own topography. The interaction between incoming reactive plasma species and the polymer is related to the incident angle of the species. Their deepest penetration occurs when the species fluxes are normal to the polymer surface but the presence of protusion and the roughness of the surface may alter their own path and the further etching of the polymer. Du K. et al. [27] gave an illustration on the impact of the roughness of the substrate on the deviation of species fluxes and the subsequent pattern 
growth. Different impact locations of the ions on the surface are distinguished: ionic impact on a flat area, on the top of the protusion, both with a normal incidence, or on the sidewall of the protusion. The two former ions have penetration length longer than that of the latter one. Such a scheme could explain the formation of the observed nanowires.

Another possibility of selectivity due to the polymer itself arises from polymer impurities or metal deposition on the surface. Polymeric materials are never pure compounds and depending on their further application different charges, either inorganic or organic are added to the polymeric matrix. In addition, depending on the plasma chamber design and on the excitation mode of the plasma, some metallic particles from the chamber walls or electrodes can be ejected and randomly included in the polymer matrix. Finally, the polymer substrate contains metallic and hard particles that modify the etch rate and the substrate pattern [10$13,15,18,19,29]$. Indeed, during the $\mathrm{O}_{2}$ plasma treatment of PMMA [12], nanoparticles such as antimony, aluminum, fluorine, sodium, potassium, silicon ... are self-generated and act as etch masks to form vertically aligned nanowires. It must be pointed out that with such a process, no control of the particle deposition is achieved. Furthermore, if a composite material is plasmatreated as for example carbon nanotubes/polydimethylsiloxane [29] its surface becomes patterned, rough and superhydrophobic. Such a behavior can be interpreted as a selective etching of PDMS that reveals the hydrophobic carbon fiber.

Polymers are heterogeneous materials not only on the chemical aspect but also on the structural property, since most of them are semicrystalline polymers. In the case of PET, the crystalline matrix when plasma-treated leads to a pattern whose decorative motives are sharper, like nanopillars, nanofibers [2] (Fig. 8).

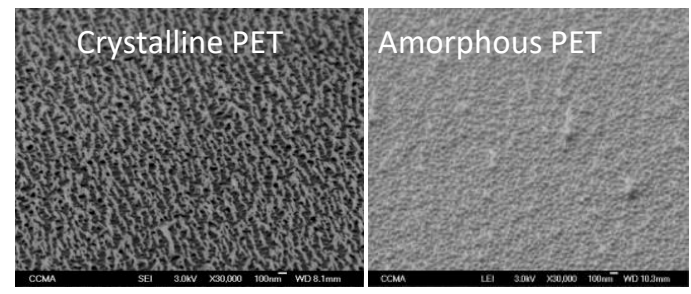

Fig. 8. Dependence of the textured plasma-treated PET on its crystallinity [2]

The crystalline domains have higher density and thus are etched with a lower rate than amorphous ones. The type of crystallites and its proportion should influence the polymer patterning as shown in the literature [2,30,31]. For amorphous polymers, the selectivity is achieved by the creation of surface crystallites through chain motion, nucleation or germination upon exposure to the plasma [2,7,9]. Differential scanning calorimetry (DSC) measurements of 
pristine and plasma treated samples have confirmed the aforementioned predictions, through the presence of crystallinity in amorphous polymers after being exposed to the plasma.

In the case of PEEK, its crystallinity rate could directly impact its ability to be textured. Indeed, the total surface / etched surface ratio is the crystallinity rate as measured by DSC and FTIR [32]. The amorphous parts of the PEEK polymer are etched before the crystalline ones leading to those features producing the global texturation (Fig. 9). Therefore, the texturing efficiency could need to take care of- or at least to have the knowledge of the surface crystallinity rate. One can argue that the process used to produce the samples may then have a great importance towards the crystallinity rate of the surface and thus towards its ability to be textured the right way depending on the application. If the surface temperature can exceed the glass transition during the plasma treatment, the atomic structure of the polymer may rearrange and its crystallinity rate can increase. This is particularly possible with an atmospheric plasma torch which supplies a higher temperature on the surface during treatment. In the case of an amorphous specimen, if processed with a high speed of cooling, the plasma treatment may produce a reorganization of the surface leading to a re-crystallization, and this has been observed up to several hundred micrometers deep under the surface. At the opposite, the polymeric crystallinity rate is not altered by the plasma torch when the pristine material is already at its maximum crystallinity rate which is about $38 \%$ for the PEEK.

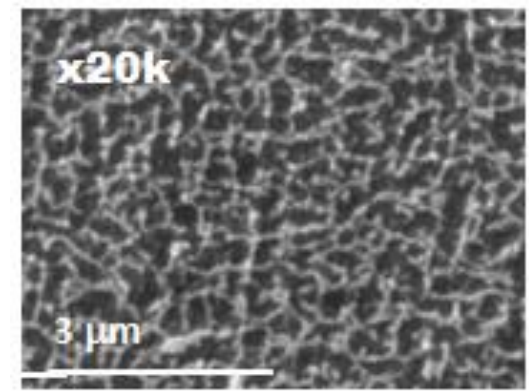

a)
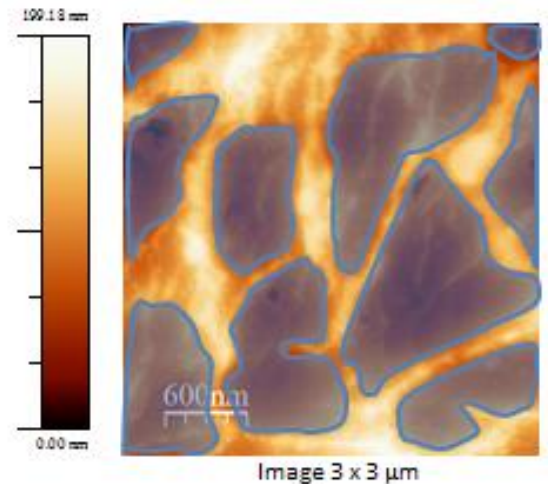

b)

Fig. 9. Texturing PEEK by the selective etching of its amorphous parts in $100 \% \mathrm{O}_{2}$ plasma and with a bias voltage of $-200 \mathrm{~V}$ a) SEM image b) AFM - Dark areas $\approx 70 \%$ of the total area were assigned to the amorphous phasesWhite upper areas $\approx 30 \%$ were assigned to the crystalline parts [32].

Polymers are stretchable materials, therefore the patterning process is also studied when polymers are stretched, specifically the PDMS, since this polymer induces a different pattern, a wrinkling one. The purpose of such experiment is associated to the incorporation of a nanoscale roughness on the wrinkle walls during the strain and then, after the strain has released 
this nano-scale feature is hidden, and finally a mechanical-responsive material is prepared [3335]. The morphology of wrinkle patterns, specifically their wavelength and amplitude is controlled by the strain release after the $\mathrm{O}_{2}$ plasma step. This can be repeated several times in order to obtain different sizes of wrinkle patterns with a wavelength in the range of $50 \mathrm{~nm}$ to $10 \mu \mathrm{m}$ and an amplitude in the range of $20-400 \mathrm{~nm}$ (Fig. 10) [33].
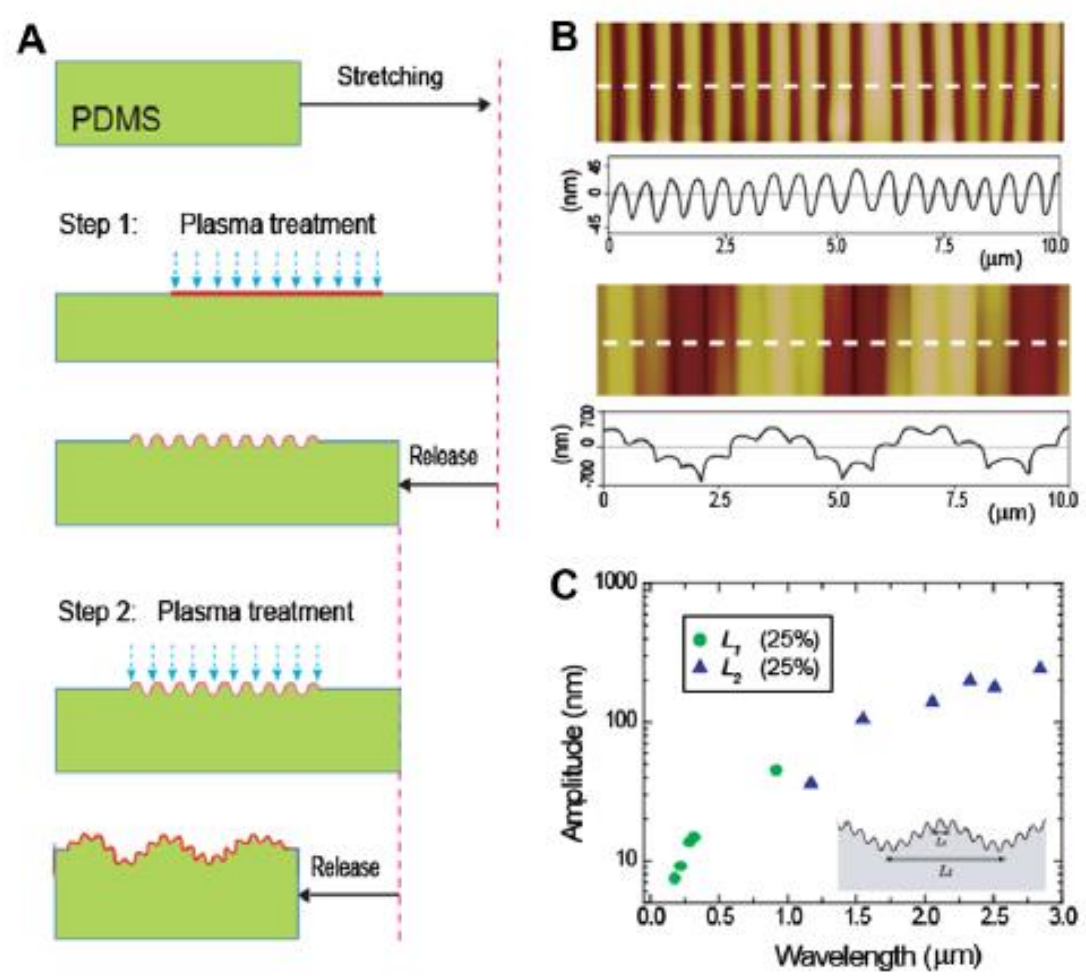

Fig. 10. PDMS multi-step plasma treatment for the creation of hierarchical wrinkles. (A) Schematics of the experiment. (B) AFM images of hierarchical wrinkle patterns created by a two-step plasma treatment [33].

All the methods described above concern plasma etching of industrial bulk polymers, while only few applications to plasma-deposited films were found [36]. The issue with plasma polymers is the absence of linear chains which makes them difficult to form crystalline domains. Thus, no selectivity of degradation is possible due to crystallization. Plasmapolyaniline films treated by argon plasma show nanostructured surfaces whose density and dimensions depend on the operating conditions. The creation of the nanodots is mainly iondependent according to the ion energy and the ion flux. In addition, when the substrate is polarized, sputtering effects become important such that sputtering and ion-induced diffusion become competitive mechanisms. It is interesting to note that some of these parameters do not have the same influence on thin film than on bulk polymers [36]. For example, for conventional polymers, the rise of processing time and of discharge power leads to increase the height of nanofibers and therefore of the surface texturation. This observation suggests that the creation of the grains on plasma-polymer surface does not have the same origin as on bulk polymer. 


\section{One-step bottom-up plasma texturing}

Over several decades, plasma polymerization of a wide range of compounds has been fully recognized as an important process for the formation of new thin layers deposited on most substrates, using a simple one-step procedure. The possibility of obtaining different morphologies of plasma polymers prepared without templates, at atmospheric pressure or at low pressure, in a continuous or pulsed mode has been studied for only few years $[7,30,31,37$ 39]. The most relevant works describe either the creation of nanoribbons, nanowires whose size is comprised between $15-20 \mathrm{~nm}$ [7,30,37] or nanodots, nanopillars [31,38,39]. Despite the consequent enhanced properties due to the patterning, these structures are not entirely normally aligned to the surface as observed with the top-down process.

However, Zaitsev et al. [38,39] demonstrate how to obtain well-defined onedimensional polymer structures while preserving the monomer chemical structure. The process (two-step or three-step process) is based on the gradual decrease of the discharge power during the deposition. A high power is first applied during a short time in order to form polymer nuclei followed by a low power step for a long time allowing the obtention of a well-ordered polymer structure. Fig. 11 illustrates the chemical structure and the morphology of the obtained plasma polymer using aniline as monomer. The polymerization of aniline at $300 \mathrm{~W}$ during $1 \mathrm{~min}$ leads to a textured surface (Fig. 11 a) but without retention of the monomer structure (Fig. 11 c, 300 $\mathrm{W})$. On the contrary, the low power $(60 \mathrm{~W})$ aniline polymerization gives a smooth surface with a low monomer fragmentation (Fig. $11 \mathrm{c}, 60 \mathrm{~W}$ ). By combining these conditions in a two-step process, plasma polyaniline presents a textured surface (Fig. $11 \mathrm{~b}$ ) with retention of the aniline structure (Fig. $11 \mathrm{c}$, middle spectrum).

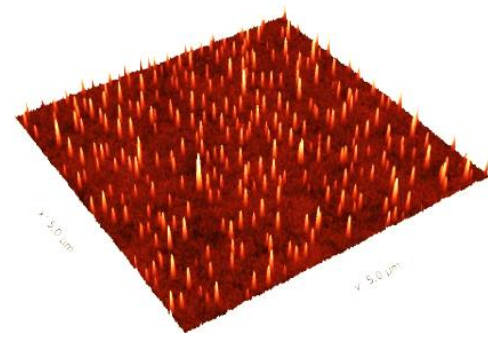

(a) 


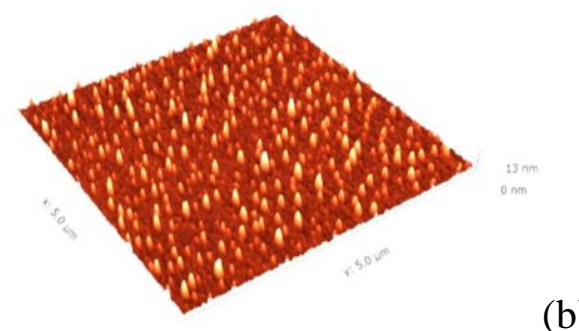

(c)

(b)

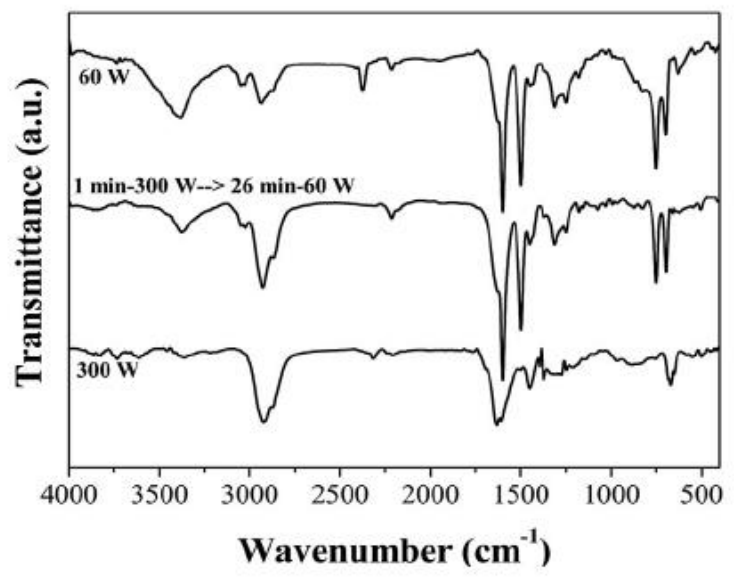

(c)

Fig. 11. Plasma polyaniline prepared in different conditions. (a, b) AFM images. (c) FTIR spectra [38].

This polymer structuring can be associated with the partial dewetting of plasma polymer chains as already observed with other coatings techniques (spin-coating, annealing of thin layers...) [40]. This dewetting namely the diffusion of reactive species on the substrate surface strongly depends on the temperature of this surface which is time and power dependent. Fig. 12 presents the dependence of the polymer roughness on the surface temperature. This figure shows that the dewetting occurs within a range of surface temperature comprised between 40 and $50^{\circ} \mathrm{C}$. In this range of temperature, the film roughness drastically increases and nanodots appear while below this temperature range, the plasma polymer is rather smooth.

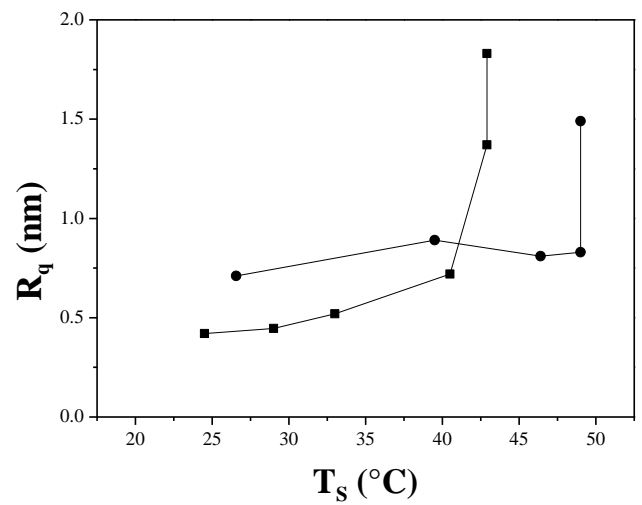

Fig. 12. Dependence of plasma polyaniline roughness Rq on surface temperature Ts [38].

Above this range, the temperature is too high and leads to a liquid-like state of the film. In this state, the film is unable to form any nanostructures and thus a smooth uniform film is obtained. Such operating conditions thus degrade chemical structure of both precursor and growing polymer and a multi-step process including low discharge power can be applied if the chemical structure of the precursor must be preserved [39]. 
Similar structures have been obtained with fluorinated plasma-polymers [7,37,41]. All single step methods combine the fluorinated chemistry with a textured surface whose elemental pattern deals with micrometer-long ribbon-shaped nanostructures rather than nanopillars under pulsed fluorinated plasmas. The ribbon-like structure is achieved at low duty cycle, long modulation period and duration [37]. The proposed growth mechanism deals with successive steps as the creation of nucleation centers, the nucleus alignment into a spiral mimic, the attachment of further spheric nuclei, and termination with further nucleation sites on the ribbon surface.

\section{Plasma texturing combined with other methods}

\subsection{Plasma texturing combined with laser irradiation}

Laser texturing followed by plasma modification or deposition offers the possibility of synthesis of hierarchically patterned surface with locally heterogeneous properties $[24,42,43]$. While the plasma treatment leads to nanotopography, the laser treatment induces microstructures over the polymeric surface as observed with laser-irradiated and plasmaoxidized PEEK. The laser treatment leads, through a thermal or a photodegradation mechanism according to the wavelength of the laser beam, to the microstructuring ablation from the surface. The formed dot arrays separated by the interspacing of several hundred interspacing in two axes are in the shape of microcraters with a diameter around $20 \mu \mathrm{m}$ and depths around $600 \mathrm{~nm}$. After the plasma treatment, the polymeric surface bears 1D nanostructures with a diameter around 30 - $40 \mathrm{~nm}$ and a length of few micrometers (Fig. 13. [42]). This dual treatment leads to a unique topography composed of both nanofibrous surface structures and microcraters.

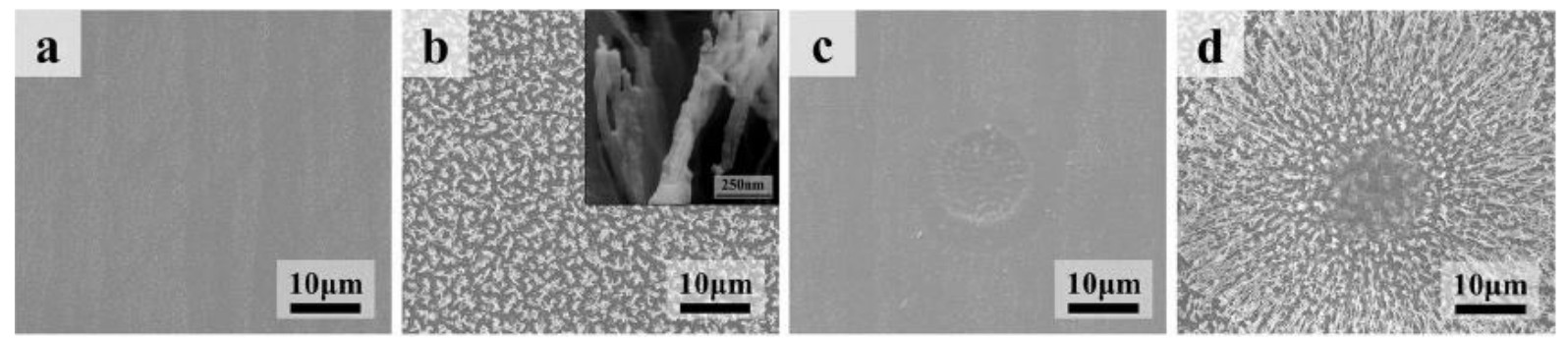

Fig. 13. SEM images of prepared surfaces: a non-treated, b plasma-treated, c laser-treated, and d plasma and laser-treated [42]

\subsection{Other combined routes of texturing}


Most of the combined techniques coming from the microelectronic background are dealing with the plasma treatment of polymeric films in the presence of masks or templates that induce the creation of the first level of the required roughness scale [34,44-46]. This review is not focused on this route. However such a process allows to prepare onto strained PDMS singular surfaces pattern, hierarchically wrinkled one with the well-defined wavelength / amplitude, organization, orientation, but also location as described in [34]. Indeed, the strained areas in contact to $\mathrm{O}_{2}$ plasma form a hard zone because of the $\mathrm{SiO}_{2}$-like layer growth while the unexposed areas remain soft zones with different mechanical behaviors after the strain releases.

Plasma deposition in presence of nanoparticles injected alone or included in an aerosol droplet in the plasma phase sometimes so-called atomized spray plasma deposition create hierarchical hybrid plasma polymer [45,47-49]. The plasma deposition of the organic precursor onto these nanoparticles suspended in gas phase allows to prepare textured hydrocarbon surface when the plasma-coated particles precipitate onto a substrate. Thus, if the initial particles have different sizes, the final surface shows a dual-scale roughness that achieves for example the superhydrophobic, surperhydrophilic property. The increase of the plasma parameters (duration, concentration of aerosol droplets) has a significant effect on the chemical and morphological structure of the thin films which ultimately leads to superhydrophobic with low contact angle hysteresis due to the hierarchical multiscale roughness of the coating. This approach brings a major advantage to coat any substrate materials including polymers or metals, which enlarges the possible range of applications of this process.

In order to produce smart materials for wide applications such as in biomimetics, hierarchical structures can also be prepared by combining the micro-imprint technique followed by plasma etching [50-52]. Two scales of roughness are obtained, the first one at the microscale thanks to the molding of photocured polymers or blends so-called negative stamps and then, the embossed film is simply exposed to an oxygen plasma to produce the hierarchical structure (Fig. 14.). A plasma-deposit could also induce the final structure.

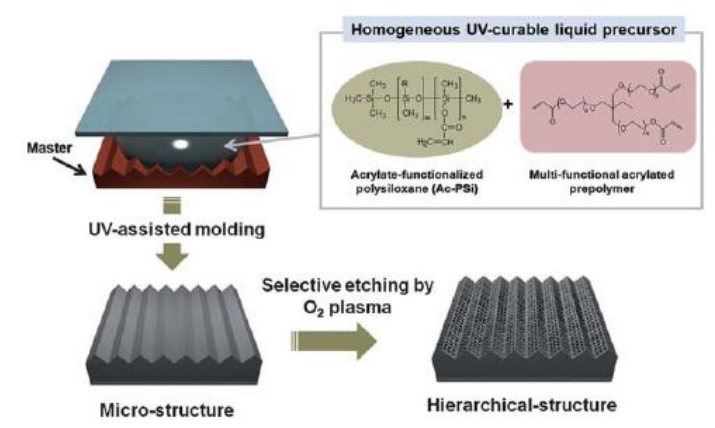

Fig. 14. Route of synthesis of hierarchical structure from imprint and plasma etching [50] 
Some examples of the colloidal lithography including the spin coating of PS nanoparticles on both silicon or polymeric substrates are illustrated in [46,53,54]. With this two-step process, the atmospheric plasma treatment induces not only the "cauliflower-like" nanotexturing of the PS spheres but also the etching of the underlying polymer (Fig. 15). After etching/nanotexturing, Ellinas K. et al. [54] gave evidences of a hierarchical triple-scale roughness with microscale ordered columns, and dual-scale (hundred nano/ten nanometers) nanoscale texture on the particles (top of the column) and on the etched PMMA surface.

The colloidal lithography has the major disadvantages that the scale of the first roughness depends on the colloid size, on the chemical nature of the commercial product and further on its availability that limits its application. The so-called breath-figure technique leads to well-organized structures bearing micropores obtained via the condensation of water vapor onto the surface of the evaporating polymeric solution. The structure and the morphology of the produced film are controlled by several factors such as temperature and relative humidity as well as chemical nature, concentration, viscosity, density, surface-free energy, and solubility parameters of the casting solution and therefore; a large variety of micropatterns of many types of polymeric materials are successfully synthesized [55]. Then, the double scale of roughness is achieved by plasma etching or deposition.

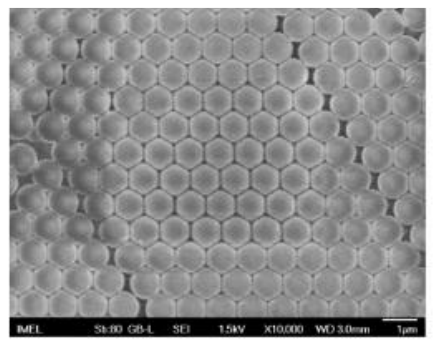

(a)

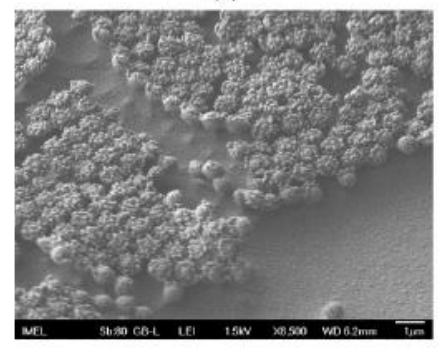

(c)

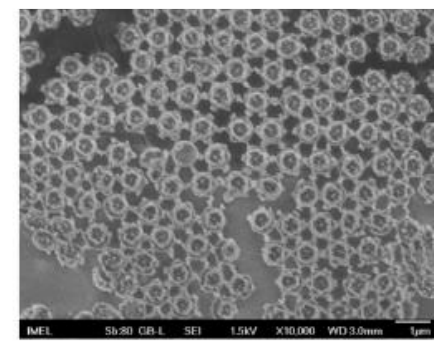

(b)

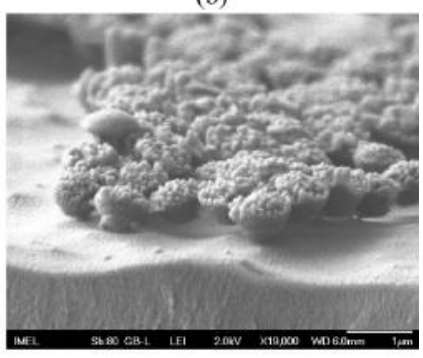

(d)

Fig. 15. SEM images of colloidal PS nanoparticles coated on PMMA and etched with atmospheric pressure $\mathrm{He} / \mathrm{O}_{2}$ plasma: (a) untreated surface $(\mathrm{d}=1 \mu \mathrm{m}$ ), (b) plasma treated surface for $8 \mathrm{~min}$, (c) plasma treated surface for $8 \mathrm{~min}$ (tilted surface), (d) plasma treated surface for $8 \mathrm{~min}$ (tilted surface at higher magnification) [46]

\section{Applications of plasma-texturing of polymers}


If the hierarchical textured surfaces whatever the chosen preparation route are also chemically functionalized, the material properties will be extended from superhydrophilic to superhydrophobic character including both Cassie-Baxter and Wenzel types depending on the pattern and the functional groups. Such singular behavior that can be summarized as the resulted effect of an increased specific surface area with a more concentrated chemical group; such behavior was explored for various application domains such as optics [2,23,25,43,53,56], electronics [45,57], sensors [3, 58-60], medical devices [2,41,61-68] and metal - polymer assemblies [24,42,44,69-74].

\subsection{Application of plasma-texturing of polymers for the optic and electronic fields}

Depending on its scale, the geometrical pattern of textured material induces broadband anti-reflective properties compared to that of plane and smooth surface. Antireflective material was prepared from nanotextured PC $[2,23]$ since the reflectance of normal incident light was reduced if the nanofeatures are not able to collapse, i.e are not too tall meaning that the plasmatreatment duration is too short. A regular pattern obtained from fluorinated plasma-treatment of polycarbonate glazing commonly used in automotive application, improves its anti-reflective property by simulating the so-called "moth eye" effect. The reflectance / transmittance measurements showed that this approach is able to increase the anti-reflective property of the polycarbonate without drastically damaging its transmittance [56]. The plasma patterning in conjunction with colloidal lithography on PS has attracted considerable attention in recent years as a means to reduce Fresnel reflection in photovoltaic solar cell materials. The resulting paraboloidal hierarchical structures suppress average reflectance to below $0.5 \%$ across a spectral range of $500-1000 \mathrm{~nm}$ [53].

Besides the direct optical behavior of patterned surfaces, the combination of both extreme wetting states to micro, nanopatterns opens up interesting applications, as the example of the fog-collecting [43], antifogging and antiicing properties [25] of materials applied in optic domains. Microfluidic channels were manufactured thanks to a three-step fabrication [43]. In the first step, a double-hierarchical superhydrophilic surface structure was generated on glass using femtosecond laser. In the second step, a superhydrophobic fluorinated plasma polymer was deposited. In the last step, the coating was selectively removed by laser ablation to uncover superhydrophilic spots. The fog-collection efficiency is due to the combination of water attraction and water repellency: the superhydrophilic areas act as drop accumulation zones, whereas the surrounding superhydrophobic regions allow a fast water transportation. The 
patterned superhydrophobic surfaces are water repellent and therefore could act as anti-icing or de-icing surfaces [25]. However, it appears that a strictly repetitive pattern is required for increasing the freezing delay time, even if depending on the polymer structure and on the plasma chemistry, sharp or rounded features are prepared. Therefore, the combined technique including nanoimprint allowing a more regular pattern increases the freezing delay time.

The combination of template-assisted self-assembly of nanocrystals with plasma polymerization allows synthesis of nanocrystals with tailoring properties explored in electronic field [57]. Same methodology was also preconized for the preparation of mechanically stable silicon anodes [45].

\subsection{Application of plasma-texturing of polymers for the sensor field}

Chemical sensors play a major role in various fields (medical diagnosis, environmental monitoring....). Improving the sensor characteristics specifically induces the detection of lower concentrations of the targeted molecules. To realize this goal, one of the proposed methods is focused on the increase of the specific surface area of the sensing layer. Indeed, these textured sensors were developed since their surface nanostructures, and associated roughness, lead to a larger surface contact and therefore to the improvement of sensor performances. As most of these sensors operate at ambient temperature, if the superhydrophobic character is added, their very low surface energy also avoids moisture adsorption while they are more attractive for organic molecules $[3,58]$.

Another example of texturing effect is given with plasma-polyaniline. Polyaniline is known to be sensitive to ammonia gas. The absorbance variation measurements (at $\lambda=430 \mathrm{~nm}$ ) can be used to characterize plasma-polyaniline under gas. Fig. 16 presents the evolution of absorbance of the polymer elaborated by the two-step process and submitted to $4618 \mathrm{ppm}$ of ammonia. First, the absorbance of the layer is constant under air and then decreases in presence of ammonia until a plateau is reached. When ammonia flux is turned off, the regeneration of the polymer begins. 


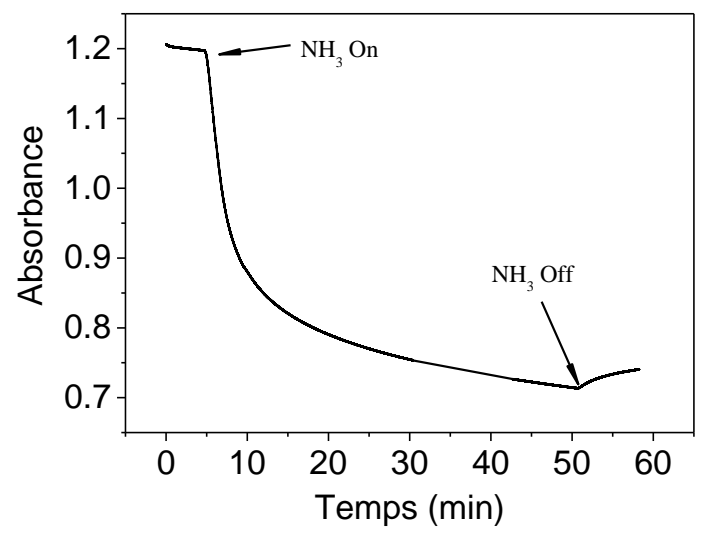

Fig. 16. The absorbance variation of plasma-polyaniline elaborated by the two-step process $(t=1 \mathrm{~min}, P=240$ $\mathrm{W}, t=26 \mathrm{~min}, P=60 \mathrm{~W}$ ) and submitted to $4618 \mathrm{ppm}$ of ammonia [59].

In the two-step process bottom-up process (Cf. §2), the duration and the power of the first step have been varied in order to study the sensitivity of the obtained layers (Fig. 17) [60]. The power and the duration of the second step are kept constant at $60 \mathrm{~W}$ and $26 \mathrm{~min}$ respectively. The horizontal line in the figure represents the sensitivity of a smooth layer elaborated in one step at $60 \mathrm{~W}$ during $26 \mathrm{~min}$, this sample is the reference. Fig. 17 shows the improvement of the gas sensitivity at the shortest first step duration regardless of the power compared to the smooth surface. Moreover, the sensitivity decreases with the rise of the power and the duration of the plasma. These results can be explained by the specific surface of the nanostructured film and by the chemical structure of the layer.

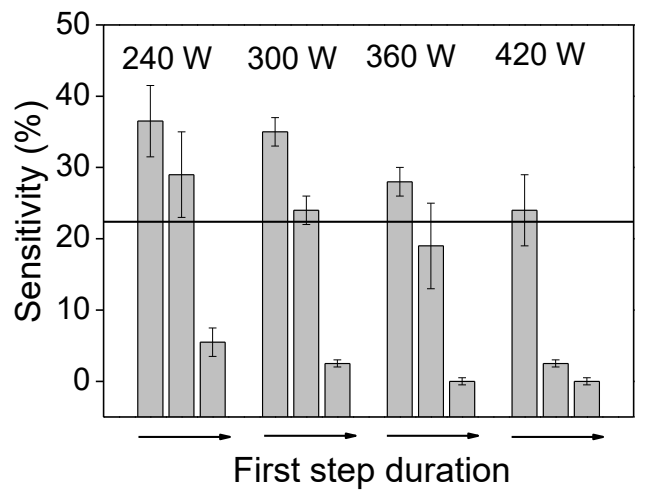

Fig. 17. Sensitivity to ammonia of plasma polyaniline layers elaborated at different powers $(P=240 \mathrm{~W}, 300 \mathrm{~W}$, $360 \mathrm{~W}$ or $420 \mathrm{~W}$ ) and deposition duration ( $t=1 \mathrm{~min}, 2 \mathrm{~min}$ or $3 \mathrm{~min}$ ) of the first step [60].

The specific surface (Table 1) of all the layers elaborated by the multi-step process is higher than that of layers synthesized in one step at $60 \mathrm{~W}$ which is $1 \mu \mathrm{m}^{2} / \mu \mathrm{m}^{2}$. The best sensitivity is obtained for layers elaborated at $240 \mathrm{~W}$ during $1 \mathrm{~min}$ for the first step. It appears that a small increase in the specific surface significantly improves the layer sensitivity to gas. 
However, the specific surface increases with power whereas ammonia sensitivity decreases. Moreover, at a given power, the sensitivity decreases with the rise of the deposition time to become, in some cases, lower than that of the smooth layer. Therefore, the chemical structure of the plasma polymer also plays an important role. The results prove that a dense layer is formed at high power or duration where the ammonia molecules can hardly diffuse, hence a decrease in sensitivity with the rise of input power or deposition duration.

Table 1. Specific surface of plasma-polyaniline elaborated by the two-step process at different duration and powers of the first step

\begin{tabular}{|c|c|c|c|c|c|c|}
\hline First step power $(\mathrm{W})$ & \multicolumn{3}{|c|}{240} & \multicolumn{3}{c|}{420} \\
\hline First step time $(\mathrm{min})$ & 1 & 2 & 3 & 1 & 2 & 3 \\
\hline Specific surface $\left(\mu \mathrm{m}^{2} / \mu \mathrm{m}^{2}\right)$ & 1.009 & 1.076 & 1.006 & 1.114 & 1.085 & 1.078 \\
\hline
\end{tabular}

\subsection{Application of plasma-texturing of polymers for the biomedical field}

Recently, hierarchical surface patterns consisting of a mixture of micro- and nano-sized structures have been investigated in terms of their effect on bioadhesion of bacteria and proteins and on cellular responses [61-63,65-68]. Thus, such surface patterning has a great academic interest for studying the biofilm formation, the competitive adsorption of proteins on surfaces with heterogeneous wettability and clarifying the relationships between protein adsorption and cell orientation, spreading and migration.

The superhydrophobic hierarchical surfaces were investigated for the purpose of antiadhesion of bacteria. However, their bio antifouling potentiality is still unclear. Indeed, Tarrade J. et al. [2] shows that whatever the surface morphology of virgin PET, plasma-fluorinated surfaces (smooth and hydrophobic, Wenzel or Cassie-Baxter superhydrophobic ones), their bacteria adhesion behavior is almost the same as compared to the untreated surface (Fig. 18). The only noticed difference is on the biofilm compacity, that formed on the two superhydrophobic PET is weaker than that of virgin and hydrophobic PET. These results were explained by mechanical anchoring of the bacteria if any pili can grow from the bacteria material as shown on SEM images (Fig. 19).

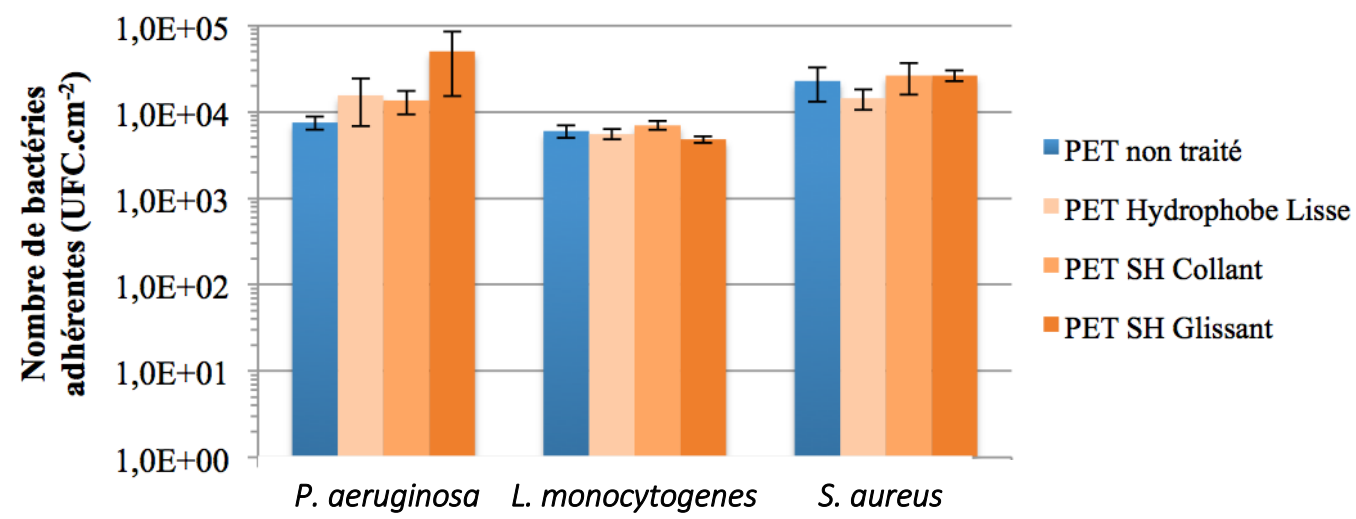


Fig. 18. Bacteria adhesion on different patterned PET surfaces [2]

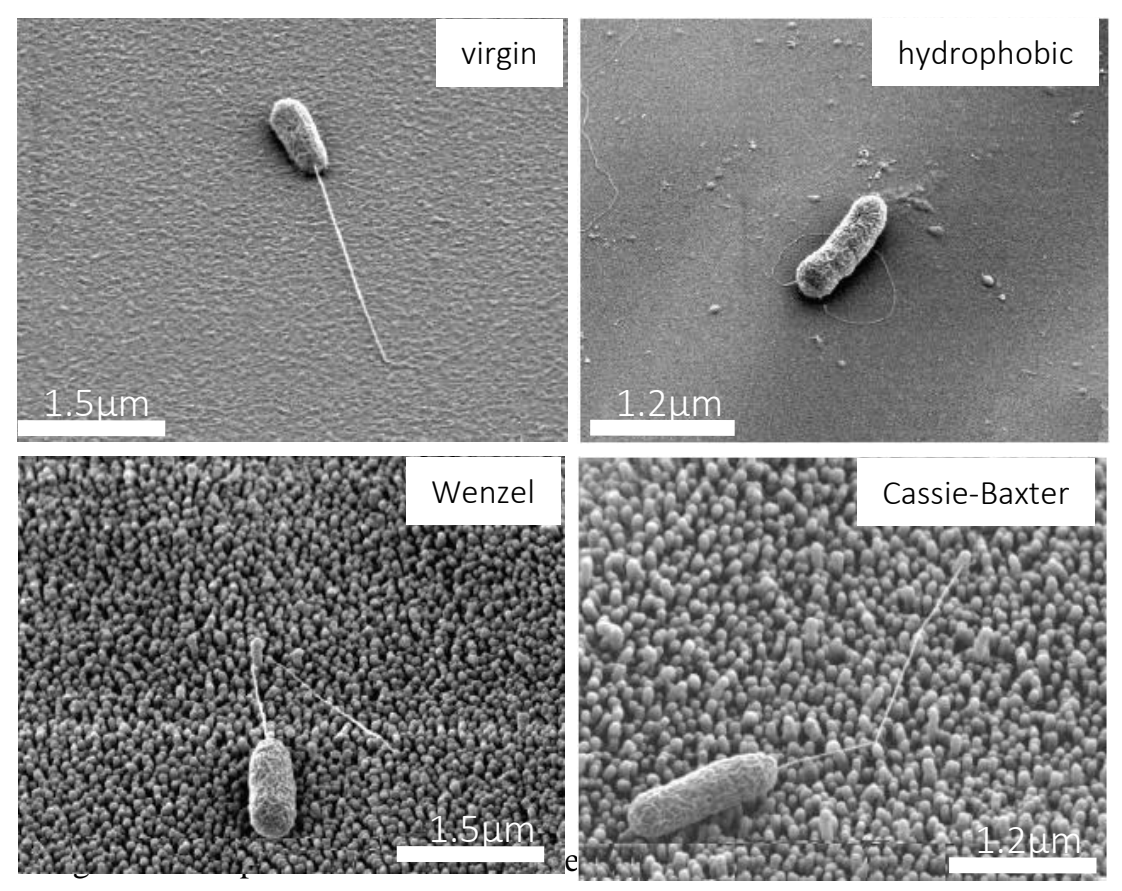

Such results are contrary to those obtained with hierarchical $\mathrm{CF}_{4}$ plasma-treated PP which exhibits a $99.6 \%$ reduction of $E$. coli adhesion compared to untreated PP [64]. Such a different behavior could be interpreted as different bacterial behavior or a different surface pattern. The PP one appears to bear less nanostructures resulting in a higher air incorporation in the polymeric matrix. In addition, superhydrophilic surfaces in contact with water spontaneously form a water monolayer that acts as a weaker boundary layer for the biomolecules adhesion as observed with POE films. But same PP plasma-oxidized and textured in a same manner as the previous one [64] and thus completely wetted with water is more adhesive for bacteria. The additional nanostructuring reduces the surface anti-biofouling properties.

Such hybrid patterns have been described being among those having the greatest influence on the proliferation and differentiation of cells. A heterogeneous polymeric surface with a regular pattern could be achieved with a partial plasma modification leading to an area whose hydrophilic or hydrophobic character is opposite to untreated zones [61]. But depending on the scale of the hierarchical roughness, the textured surface may not match ideally the nanostructure off cell adhesion complexes. Therefore, this requires the fine-tuning of nanoarchitecture of plasma layers for the biomolecule immobilization and cell adhesion [65] since the cell adhesion and growth vary in function of the roughness of coatings [41]. Patterning should have a broader definition extended to the microporosity. Indeed, when the microporosity 
comes from the electrospinning while the nanoroughness is obtained thanks to the plasmatreatment of the polymeric fiber, such patterned microfibers enhance dramatically the oblast cell attachment [63].

\subsection{Application of plasma-texturing of polymers for the metal adhesion}

For texturing, laser is a very well-known technology which gives rise to a well-defined and large-scale texture that can be applied to polymers in order to allow or improve the adhesion of metal coatings [70-75]. When PEEK surface was textured by an infrared $1026 \mathrm{~nm}$ radiation (224 femtosecond impulse), a pattern made of regular triangles with 60 micrometers side length induces one of the most efficient increase in adhesion for an aluminum or a copper thin film [74]. Such a laser etching can extend the practical adhesion strength of a metal onto a polymer greater than the best conditions found with an $\mathrm{O}_{2}$ ECR plasma. Obviously, those processes are not comparable in terms of surface mechanisms. The first one being mainly an anchoring mechanism (laser texturing) while the second one is assumed to be mainly a chemical mechanism (ECR plasma without extra bias voltage applied to the sample - called low-energy ECR plasma). These conditions of plasma texturing with a vacuum ECR plasma were already more effective in terms of adhesion than those provided with an air plasma torch which nevertheless enhanced the metal adhesion (Fig. 20).
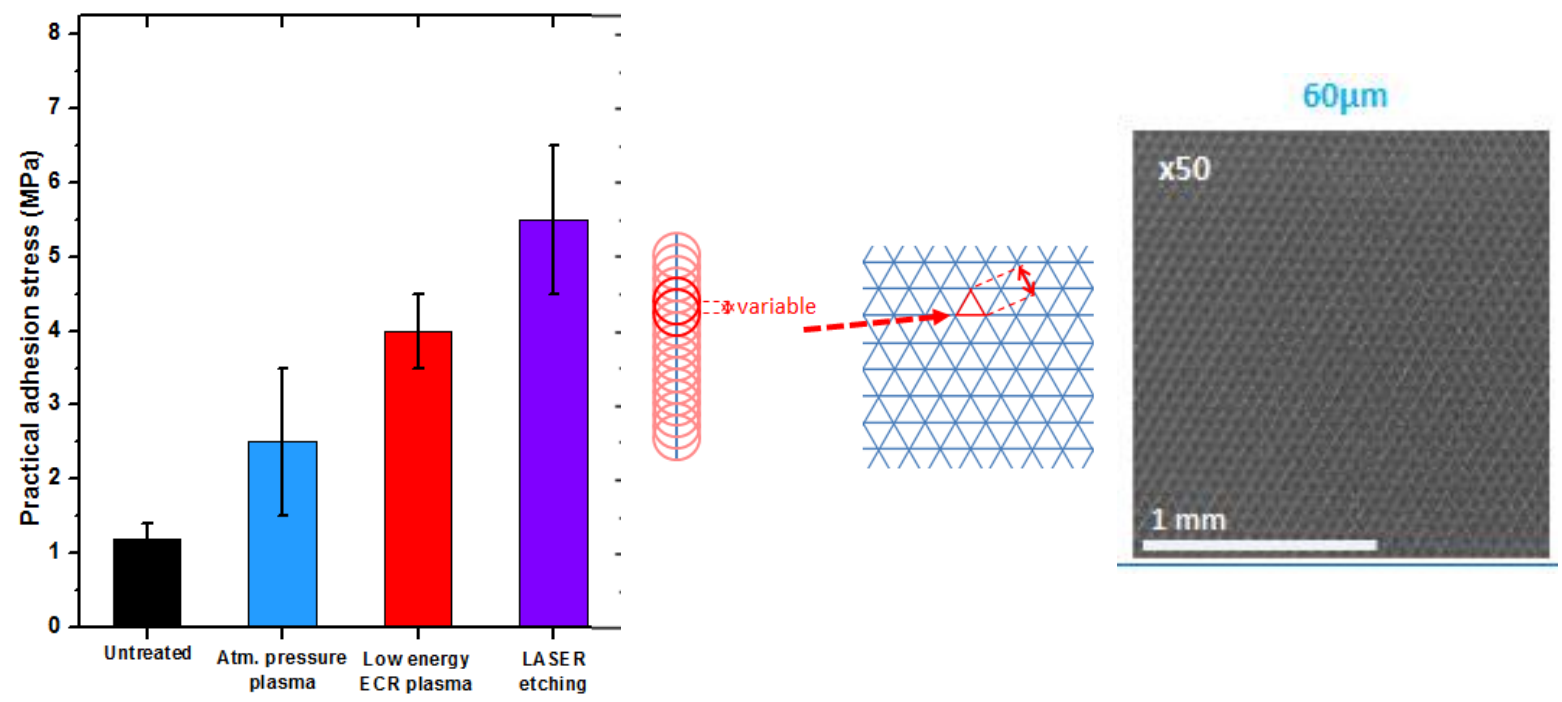

Fig. 20. Improvement of the adhesion of a metal thin film deposited on PEEK after the surface has been laser textured in comparison with plasma modified PEEK surfaces and with the untreated sample (left) and the features of the laser spot line and geometry which was selected (right) [75].

Currently, the laser ablation process leads to a two-scale alteration of the polymer (Fig 21). Inside the etched trenches, a random and small-scale texture appears to be assigned to 
polymer redeposits. These redeposits were considered sufficiently cohesive to the polymer bulk as they were not removed by the subsequent alcohol rinsing of the samples before metal deposition. These redeposit could significantly take part to the enhancement of the adhesion. By the way, the rupture of the interface when the metal-polymer assembly was mechanically tested is mainly cohesive. The rupture strength of the coating did not reach the value which is calculated if one considers the specific surface increase supplied by the triangles pattern creation. The polymer redeposits are thus assumed to limit the enhancement of the adhesion which could have been reached otherwise.

a)

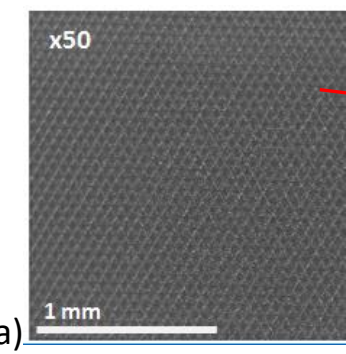

b)

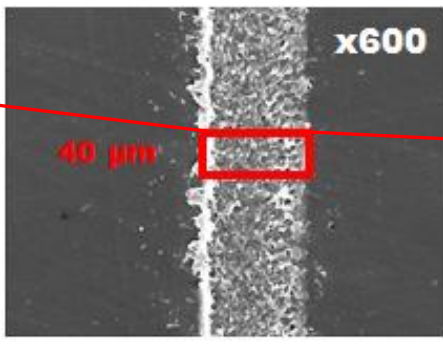

c)

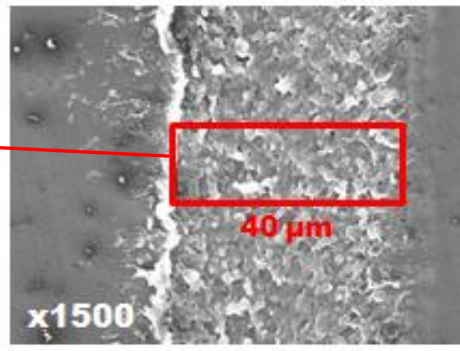

Fig. 21. SEM images of femtosecond impulse laser-treated PEEK surface showing a) first scale and b) c) second scale of texturing [75]

Even if $\mathrm{Yu} \mathrm{J.} \mathrm{et} \mathrm{al.} \mathrm{[69]} \mathrm{shows} \mathrm{that} \mathrm{the} \mathrm{surface} \mathrm{roughness} \mathrm{significantly} \mathrm{affects} \mathrm{the}$ performance of gecko mimetic adhesives, the dual modification (plasma and laser combined treatments) allows to enhance the $\mathrm{Al}$ adhesion [24,42].

Indeed, the double scale roughness positively impact the mechanical anchoring of deposited metal layer. However, this assessment is only verified if the metallic layer is perfectly covers the undulating surface topography as shown in [42] since the roughness values of the two-step modified surfaces before and after the deposition remain very close to each other. Besides the possible reinforcement of the mechanical anchoring, the chemical bonding must be taken into account. Gravis et al. [24] studied the competitive effect of the roughness and the surface chemistry on PEEK modified either by plasma (at atmospheric pressure or at reduced pressure) or by a laser beam on $\mathrm{Al}$ adhesion. Currently, the surface chemistry of PEEK can be tuned in terms of hydrophilic bonds (mainly $\mathrm{C}=\mathrm{O}$ and $\mathrm{COO}$ ) leading to an increase of its surface-free energy therefore leading to an increase of the adhesion between a metal coating and the polymer (Fig. 22). An ECR plasma treatment can add a texturing effect to the polymer surfaces especially if an external bias voltage is applied to the samples (called high energy ECR). In that case, one can overlay the advantage of the chemical functionalization of the surface to the advantage of the anchoring effect to extend the result in terms of adhesion as shown on Fig 22. 

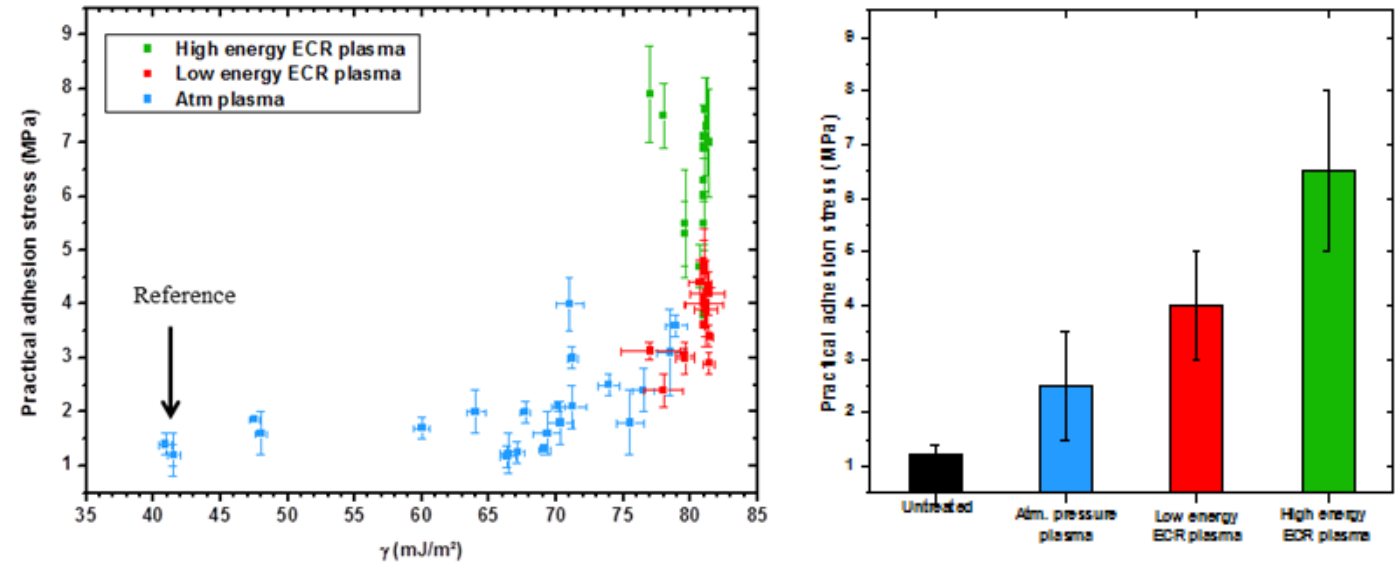

Fig. 22. Comparison between an atmospheric plasma, a low energy ECR plasma and a high energy ECR plasma treatment on the surface-free energy $(\square)$ and on the practical adhesion on PEEK surfaces. [24]

In conclusion, on polymer-metal adhesion, Fig. 23 provides an overview on the balance that one can select between the chemical (functionalization) and the texturing (mechanical anchoring) effects on a polymer surface as supplied by plasma and laser technologies. Polymer texturing is playing a major role towards the anchoring effect which can be filled out with the chemical modification of the surface with only one process in the case of plasmas.

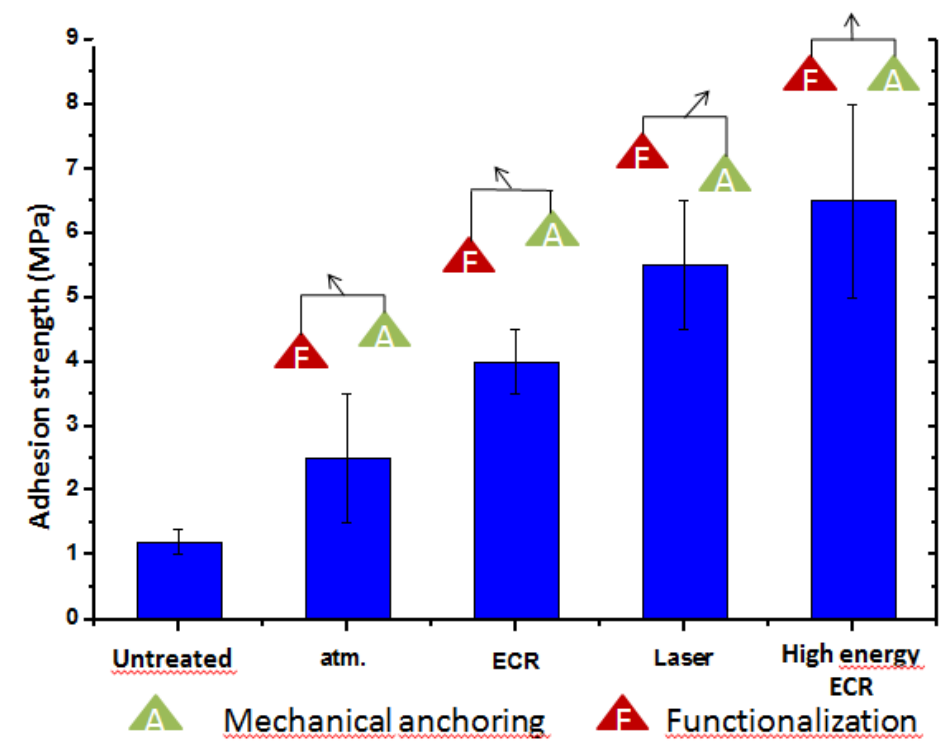

Fig. 23. Competitive balance between mechanical anchoring and surface chemistry of PEEK in the case of different plasma treatments or laser texturing

Surface patterning for adhesion purpose is also developed for the metallic ink used in inkjet printing; the aim is not only to improve the adhesion of metallic ink but to limit its spreading during the printing (Fig. 24). 


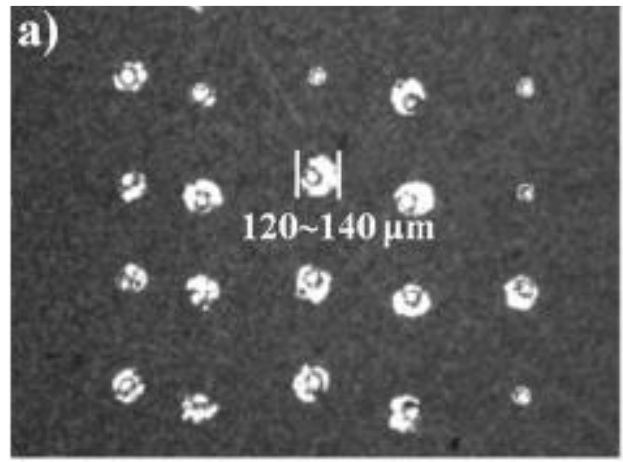

$<$ As-Is PI film>

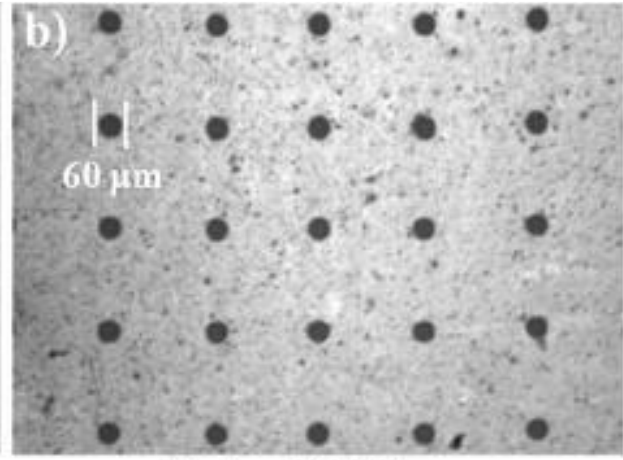

$<$ Textured PI film>

Fig. 24. Optical microscope images of Ag dot pattern formed by an inkjet printing system on the polyimide surface before (a) and after (b) texturing using atmospheric pressure plasmas [44].

That means to balance the hydrophilic / hydrophobic character of the substrate and to control the wettability equilibrium. For such a purpose [44], a multiple-step plasma treatment including deposition, oxidation and etching of a polyimide surface leads to micro-patterned surface with hydrophilic and superhydrophobic Wenzel-type domains. Such a treated surface bears fine lines of adhesive Ag ink.

\section{Conclusion}

The aim of this review was to illustrate the versatility of plasma nano-texturing. Nanofeatures with high aspect ratio can be prepared either by etching or deposition whatever the type of polymer and plasma. Even more, a regular pattern is obtained without any mask layered on the polymeric substrate. Furthermore, plasma nano-texturing has a low environmental impact, it can be combined to other dry technics. The texturing mechanism was illustrated through different applications.

\section{References}

1. Control of nanotexture and wetting properties of polydimethylsiloxane from very hydrophobic to super-hydrophobic by plasma processing, K. Tsougeni, A. Tserepi, G. Boulousis, V. Constantoudis, E. Gogolides, Plasma Process. Polym. 4 (2007) 398-405.

2. Texturation and superhydrophobicity of polyethylene terephthalate thanks to plasma technology, J. Tarrade, Th. Darmanin, E. Taffin de Givenchy, F. Guittard, D. Debarnot, F. Poncin-Epaillard, Appl. Surf. Sci. 292 (2014) 782-89.

3. Tunable poly(dimethylsiloxane) topography in $\mathrm{O}_{2}$ or Ar plasmas for controlling surface wetting properties and their ageing, K. Tsougeni, A. Tserepi, G. Boulousis, V. Constantoudis, E. Gogolides, Jpn. J. Appl. Phys. 46 (2007) 744-750.

4. Ultrahydrophobic PMMA micro- and nano-textured surfaces fabricated by optical lithography and plasma etching for X-ray diffraction studies, A. Accardo, F. Gentile, F. 
Mecarini, F. De Angelis, M. Burghammer, E. Di Fabrizio, C. Riekel, Microelectron. Eng. 88 (2011) 1660-1663.

5. Hierarchical, plasma nanotextured, robust superamphiphobic polymeric surfaces structurally stabilized through a wetting-drying cycle, AK. Gnanappa, DP. Papageorgiou, E. Gogolides, A. Tserepi, AG. Papathanasiou, AG. Boudouvis, Plasma Process. Polym. 9 (2012) 304-315.

6. Superhydrophobic, hierarchical, plasma-nanotextured polymeric microchannels sustaining high-pressure flows, DP. Papageorgiou, K. Tsougeni, A. Tserepi, E. Gogolides, Microfluid Nanofluid 14 (2013) 247-255.

7. Plasma nanostructuring of polymers: different routes to superhydrophobicity, A. Milella, R. Di Mundo, F. Palumbo, P. Favia, F. Fracassi, R. d'Agostino, Plasma Process. Polym. 6 (2009) 460-466.

8. Fluorocarbon plasmas for nanotexturing of polymers: A route to water-repellent antireflective surfaces, R. Di Mundo, V. De Benedictis, F. Palumbo, R. d'Agostino, Appl. Surf. Sci. 255 (2009) 5461-5465.

9. Nanofibrillar patterns by plasma etching: the influence of polymer crystallinity

and orientation in surface morphology, E. Wohlfart, JP. Fernández-Blázquez, E. Knoche, A. Bello, E. Pérez, E. Arzt, A. del Campo, Macromolecules 43 (2010) 908-9917.

10. Nanostructures formed on carbon based materials with different levels of crystallinity using oxygen plasma treatment, TJ. Ko, W. Jo, HJ. Lee, K.H. Oh, MW. Moon, Thin Solid Films 590 (2015) 324-329.

11. Self-masked high-aspect-ratio polymer nanopillars, MH. Chen, YJ. Chuang, FG. Tseng, Nanotechnology 19 (2008) 505301.

12. Fabrication of polymer nanowires via maskless $\mathrm{O}_{2}$ plasma etching, K. Du, I. Wathuthanthri, Y. Liu, YT. Kang, CH. Choi1, Nanotechnology 25 (2014) 165301.

13. Plasma micro-nanotextured, scratch, water and hexadecane resistant, superhydrophobic, and superamphiphobic polymeric surfaces with perfluorinated monolayers, K. Ellinas, SP. Pujari, DA. Dragatogiannis, CA. Charitidis, A. Tserepi, H. Zuilhof, E. Gogolides, ACS Appl. Mater. Interfaces 6 (2014) 6510-6524.

14. Morphological and chemical effects of plasma treatment with oxygen $\left(\mathrm{O}_{2}\right)$ and sulfur hexafluoride $\left(\mathrm{SF}_{6}\right)$ on cellulose surface, JSG. de Camargo, A. de Menezes, NC. da Cruz, EC. Rangel, A. de Oliveira Delgado-Silva, Mat. Research. 20(Suppl. 2) (2017) 842-850.

15. Easy plasma nano-texturing of PTFE surface: From pyramid to unusual spherules-onpyramid features, C. Lo Porto, R. Di Mundo, V. Veronico, I. Trizio, G. Barucca, F. Palumbo, Appl. Surf. Sci. 483 (2019) 60-68.

16. Elaboration of nano-structured grafted polymeric surface, T. Vrlinic, D. Debarnot, M. Mozetic, A. Vesel, J. Kovac, A. Coudreuse, G. Legeay, F. Poncin-Epaillard, J. Colloid Interface Sci. 362 (2011) 300-310.

17. Mechanisms of oxygen plasma nanotexturing of organic polymer surfaces: from stable super hydrophilic to super hydrophobic surfaces, K. Tsougeni, N. Vourdas, A. Tserepi, E. Gogolides, C. Cardinaud, Langmuir 25 (2009) 11748-11759.

18. Controlled growth of aligned polymer nanowires, H. Fang, W. Wu, J. Song, Z.L. Wang, J. Phys. Chem. C 113 (2009) 16571-16574.

19. Plasma nano-texturing of polymers for wettability control: why, what and how, F. Palumbo, C. Lo Porto, P. Favia, Coatings 9 (2019) 640.

20. Manipulation of the superhydrophobicity of plasma-etched polymer nanostructures, K. Du, Y. Jiang, Y. Liu, I. Wathuthanthri, CH. Choi, Micromachines 9 (2018) 304.

21. Thermal annealing as a new simple method for PTFE texturing, P. Glaris, JF. Coulon, M. Dorget, F. Poncin-Epaillard, Polymer 54 (2013) 5858-5864. 
22. Topographical length scales of hierarchical superhydrophobic surfaces, PK. Dhillon, PS. Brown, CD. Bain, JP. Badyal, S. Sarkar, Appl. Surf. Sci. 317 (2014) 1068-1074.

23. Nano-texturing of transparent polymers with plasma etching: tailoring topography for a low reflectivity, R. Di Mundo, M. Troia, F. Palumbo, M. Trotta, R. d'Agostino, Plasma Process. Polym. 9 (2012) 947-954.

24. Etude de l'influence de la physicochimie et de la texturation de surface sur l'adhérence métal - poly(ether ether ketone) (PEEK), D. Gravis, PhD, Le Mans Université, mars 2019.

25. Hydrophobicity and anti-icing performances of nanoimprinted and roughened fluoropolymers films under overcooled temperature, J. Durret, N. Frolet, C. Gourgon, Microelectron. Eng. 155 (2016) 1-6.

26. Nanotextured shrink wrap superhydrophobic surfaces by argon plasma etching, JM. Nokes, H. Sharma, R. Tu, MY. Kim, M. Chu, A. Siddiqui, M. Khine, Materials 9 (2016) 196.

27. Self-formation of polymer nanostructures in plasma etching: mechanisms and applications, K. Du, Y. Jiang, PS. Huang, J. Ding, T. Gao, CH. Choi, J. Micromech. Microeng. 28 (2018) 014006.

28. Effets de la texturation couplée à la fonctionnalisation par plasma sur l'adhérence de revêtements d'aluminium sur du poly-éther-éther-cétone, G. Rigole, F. Poncin-Epaillard, JF. Coulon, 20èmes Journées d'étude sur l'Adhésion, novembre, Annecy (France)

29. Plasma-jet-assisted maskless, erasable, extreme wetting-contrast patterning on stretchable carbon nanotubes film, J. Su, Y. Jiang, D. Ye, YA. Huang, Appl. Phys. Express 12 (2019) 115503.

30. Nanofibrous polyaniline thin film prepared by plasma-induced polymerization technique for detection of $\mathrm{NO}_{2}$ gas, A. Tiwaria, R. Kumar, M. Prabaharan, RR. Pandey, P. Kumari, A. Chaturvedi, A.K. Mishra, Polym. Adv. Technol. 21 (2010) 615-620.

31. Nanostructured polyaniline thin films prepared by plasma polymerization at atmospheric pressure, HR. Humud, TK. Aubais, Phys. Sci. Res. Int. 1(4) (2013) 110-122.

32. Increasing the practical adhesion by tuning the surface physicochemistry of poly(ether ether ketone), J.-F. Coulon, D. Gravis, W. Knapp, F. Poncin-Epaillard, Euromat 2019, septembre 2019 Stockholm (Suède)

33. Surface modification of polymers using a multi-step plasma treatment, MW. Moon, A. Vaziri, Scripta Mat. 60 (2009) 44-47.

34. Controlled free edge effects in surface wrinkling via combination of external straining and selective $\mathrm{O}_{2}$ plasma exposure, Y. Yang, X. Han, W. Ding, S. Jiang, Y. Cao, C. Lu, Langmuir 29 (2013) 7170-7177.

35. Fabrication of controlled hierarchical wrinkle structures on polydimethylsiloxane via onestep $\mathrm{C}_{4} \mathrm{~F}_{8}$ plasma treatment, L. Miao, XL Cheng, H. Chen, Y. Song, H. Guo, J. Zhang, X. Chen, H. Zhang, J. Micromech. Microeng. 28 (2018) 015007.

36. Nanotexturing of plasma-polymer thin films using argon plasma treatment, A. Zaitsev, A. Lacoste, F. Poncin-Epaillard, A. Bès, D. Debarnot, Surf. Coat. Technol. 330 (2017) 196-203.

37. Morphological and structural study of plasma deposited fluorocarbon films at different thicknesses, G. Cicala, A. Milella, F. Palumbo, P. Favia, R. d'Agostino, Diam. Rel. Mater. 12 (2003) 2020-2025.

38. A bottom-up and templateless process for the elaboration of plasma-polymer nanostructures, A. Zaitsev, F. Poncin-Epaillard, A. Lacoste, D. Debarnot, Plasma Process. Polym. 13 (2016) 227-235.

39. A multi-step cold plasma process for fine-tuning of polymer nanostructuring, A. Zaitsev, F. Poncin-Epaillard, A. Lacoste, A. Kassiba, D. Debarnot, Prog. Org. Coat. 128 (2019) 112-119. 40. Growth and alignment of thin film organic single crystals from dewetting patterns, JN. Tisserant, G. Wicht, O. F. Gobel, E. Bocek, G. Bona, T. Geiger, R. Hany, R. Mezzenga, S. Partel, P. Schmid, WB. Schweizer, J. Heier, ACS Nano 7 (2013) 5506-5513. 
41. Increasing cell adhesion on plasma deposited fluorocarbon coatings by changing the surface topography, R. Gristina, E. D’Aloia, GS. Senesi, A. Milella, M. Nardulli, E. Sardella, P. Favia, R. d'Agostino, Appl. Biomater. 88 (2009) 139-150.

42. Surface topography and wetting modifications of PEEK for implant applications, CK. Akkan, ME. Hammadeh, A. May, HW. Park, H. Abdul-Khaliq, T. Strunskus, OC. Aktas, Lasers Med Sci 29 (2014) 1633-1639.

43. Fabrication of biomimetic fog-collecting superhydrophilic-superhydrophobic surface micropatterns using femtosecond lasers, E. Kostal, S. Stroj, S. Kasemann, V. Matylitsky, M. Domke, Langmuir 34 (2018) 2933-2941.

44. Polymer surface texturing for direct inkjet patterning by atmospheric pressure plasma treatment, JB. Park, JY. Choi, SH. Lee, YS. Song, GY. Yeom, Soft Matter 8 (2012) 5020.

45. A three-dimensional hierarchical structure of cyclized-PAN/Si/Ni for mechanically stable silicon anodes, W. Bao, J. Wang, S. Chen, W. Li, Y. Su, F. Wu, G. Tan, J. Lu, J. Mater. Chem. A 5 (2017) 24667-24676.

46. Atmospheric plasma etching of polymers: A palette of applications in cleaning/ashing, pattern formation, nanotexturing and superhydrophobic surface fabrication, P. Dimitrakellis, E. Gogolides, Microelectronic Engineering 194 (2018) 109-115.

47. Superhydrophobic fluorine-free hierarchical coatings produced by vacuum based method, M. Petr, J. Hanus, O. Kylian n, J. Kratochvíl, P. Solar, D. Slavínska, H. Biederman, Materials Letters 167 (2016) 30-33.

48. Aerosol-assisted atmospheric cold plasma deposition and characterization of superhydrophobic organic-inorganic nanocomposite thin films, F. Fanelli, AM. Mastrangelo, F. Fracassi, Langmuir 30 (2014) 857-865.

49. Atomised spray plasma deposition of hierarchical superhydrophobic nanocomposite surfaces, I. Castaneda-Montes, AW. Ritchie, JPS. Badyal, Colloids Surf. A 558 (2018) 192199.

50. Fabrication of a hierarchical structure by oxygen plasma etching of a photocured microstructure containing a silicon moiety, SJ. Choi, MK. Choi, D. Tahk, H. Yoon, J. Mater. Chem. 21 (2011) 14936.

51. Superhydrophobic surface structures in thermoplastic polymers by interference lithography and thermal imprinting, CWJ. Berendsen, M. Skeren, D. Najdek, F. Cerny, Appl. Surf. Sci. 255 (2009) 9305-9310.

52. Robust technique allowing manufacturing superoleophobic surfaces, E. Bormashenko, R. Grynyov, G. Chaniel, H. Taitelbaum, Y. Bormashenko, Appl. Surf. Sci. 270 (2013) 98-103.

53. Fabrication of hierarchical anti-reflective structures using polystyrenesphere lithography on an as-cut p-Si substrate, YY. Chou, KT. Lee, YC. Lee, Appl. Surf. Sci. 377 (2016) 81-85.

54. From superamphiphobic to amphiphilic polymeric surfaces with ordered hierarchical roughness fabricated with colloidal lithography and plasma nanotexturing, K. Ellinas, A. Tserepi, E. Gogolides, Langmuir 27 (2011) 3960-3969.

55. Elaboration and surface modification of structured poly(L-lactic acid) thin film on various substrates, F. Poncin-Epaillard, O. Shavdina, D. Debarnot, Mater. Sci. Eng. C 33 (2013) 25262533.

56. Low pressure plasma modified polycarbonate: A transparent, lowreflective and scratch resistant material for automotive applications, N. De Vietroa, L. Belforte, V. Guido Lambertini, F. Fracassi, Appl. Surf. Sci. 307 (2014) 698-703.

57. Plasma within templates: molding flexible nanocrystal solids into multifunctional architectures, A. Ghadimi, L. Cademartiri, U. Kamp, G. A. Ozin, Nano Letters 7(12) (2007) 3864-3868. 
58. High sensitive detection of volatile organic compounds using superhydrophobic quartz crystal microbalance, N. Andreeva, T. Ishizaki, P. Baroch, N. Saito, Sens. Actuators B 164 (2012) 15-21.

59. Exploration de la voie plasma pour la synthèse de nanostructures et de nanocomposites à base de polyaniline, A. Zaitsev, PhD, Le Mans Université, octobre 2015

60. A multi-step cold plasma process for fine-tuning of polymer nanostructuring, A. Zaitsev, F. Poncin-Epaillard, A. Lacoste, A. Kassiba, D. Debarnot, Progress Org. Coat., 128 (2019) $112-$ 119

61. Orientation of cell adhesion and growth on patterned heterogeneous polystyrene surface, E. Detrait, J.-B. Lhoest, B. Knoops, P. Bertrand, Ph. van den Bosch de Aguilar, J. Neurosci. Methods 84 (1998) 193-204.

62. Nano-texturing of poly(methyl methacrylate) polymer using plasma processes and applications in wetting control and protein adsorption, K. Tsougeni, PS. Petrou, A. Tserepi, SE. Kakabakos, E. Gogolides, Microelectron. Engin. 86 (2009) 1424-1427.

63. Nano-sized surface patterns on electrospun microfibers fabricated using a modified plasma process for enhancing initial cellular activities, HJ. Jeon, H. Lee, GH. Kim, Plasma Process. Polym. 11 (2014) 142-148.

64. Stable lotus leaf-inspired hierarchical, fluorinated polypropylene surfaces for reduced bacterial adhesion, MI. Kayes, AJ. Galante, NA. Stella, S. Haghanifar, RMQ. Shanks, PW. Leu, React. Funct. Polym. 128 (2018) 40-46.

65. Direct covalent coupling of proteins to nanostructured plasma polymers: a route to tunable cell adhesion, I. Melnichuk, A. Choukourov, Ma. Bilek, A. Weiss, M. Vandrovcova, L. Bacakova, J. Hanus, J. Kousal, A. Shelemin, P. Solar, D. Slavínska, H. Biederman, Appl. Surf. Sci. 351 (2015) 537-545.

66. Plasma nanotextured polymeric surfaces for controlling cell attachment and proliferation: A short review, A. Tserepi, E. Gogolides, A. Bourkoula, A. Kanioura, G. Kokkoris, PS. Petrou, SE. Kakabakos, Plasma Chem Plasma Process 36 (2016) 107-120.

67. Enhanced antibacterial property and osteo-differentiation activity on plasma treated porous polyetheretherketone with hierarchical micro/nano-topography, S. Wang, Y. Deng, L. Yang, X. Shi, W. Yang, ZG. Chen, J. Biomat. Sci., Polym. Ed. 29(5) (2018) 520-542.

68. Fabrication of a biomimetic hierarchical surface replicated from a lotus leaf and in vitro cellular activities, JY. Lee, H. Lee, YB. Kim, GH. Kim, Plasma Process. Polym. 12 (2015) 141152.

69. Friction and adhesion of gecko-inspired PDMS flaps on rough surfaces, J. Yu, S. Chary, S. Das, J. Tamelier, KL. Turner, JN. Israelachvili, Langmuir 28 (2012) 11527-11534.

70. Laser surface patterning to enhance adhesion of plasma sprayed coatings, R. Kromer, S. Costil, J. Cormier, D. Courapied, L. Berthe, P. Peyre, M. Boustie, Surf. Coat. Tech., 278 (2015) 171-182.

71. Evaluation of mechanical interlock effect on adhesion strength of polymer-metal interfaces using micro-patterned surface topography, WS. Kim, IH. Yun, JJ. Lee, H.-T. Jung, Int. J. Adhes. Adhes. 30 (2010) 408-417.

72. Nanostructuring of a polymeric substrate with well-defined nanometer-scale topography and tailored surface wettability, W. Lee, MK. Jin, WC. Yoo, JK. Lee, Langmuir. 20 (2004) 7665-7669.

73. Ultra-short pulsed laser ablation of polymers, AA. Serafetinides, MI. Makropoulou, CD. Skordoulis, AK. Kar, Appl. Surf. Sci. 180 (2001) 42-56.

74. Influence of structuring by laser and plasma torch on the adhesion of metallic films on thermoplastic substrates, W. Knapp, JF. Coulon, R. Grunchec, D. Djomani, Phys. Procedia, 56 (2014) 791-800. 
75. Etude de l'adhésion de couches minces métalliques sur PEEK : effet de la topographie créée par texturation laser, D Gravis, W Knapp, F. Poncin-Epaillard, JF Coulon, JADH'17Journées d'études sur l'adhésion- 2017, Ste Maxime. 